\title{
El textil como matriz para el desarrollo de las artes plásticas en los Andes
}

\author{
Sophie DesRosiers \\ École des Hautes Études en Sciences Sociales-Centre de Recherches Historiques (UMR5885) (Francia) \\ sophie.desrosiers@ehess.fr
}

Recibido: 17 de julio de 2013

Aceptado: 16 de septiembre de 2013

\section{RESUMEN}

El origen textil de numerosos diseños de tendencia geométrica presentes en varias categorías de obras de arte ha sido ampliamente comentado para algunas culturas prehispánicas de la costa peruana, pero las relaciones directas entre diseños y textiles nunca han sido estudiadas con atención. La autora demuestra cómo algunas prácticas de tejido actuales de las tierras altas andinas ayudan a leer esos diseños y a reconstruir las técnicas de cara de urdimbre en el origen de su creación. Practicadas en las tierras altas al menos desde el Horizonte Temprano, esas técnicas no son una invención reciente, a pesar del número limitado de ejemplos conservados. Las numerosas reproducciones de sus diseños con otras técnicas textiles -bordado y tapiz entre otros- o con otros materiales -pintura sobre cerámica, murales, madera esculpida- realizadas en la Costa Sur y en la Costa Central del Perú a finales del Horizonte Temprano y durante el Intermedio Temprano, ofrecen un nuevo medio para reconstruir su pasado desbaratado y para entender las relaciones entre costa y sierra en periodos durante los cuales no es fácil indagar sobre ellas. Esos textiles cara de urdimbre han jugado efectivamente un papel importante en la historia del arte andino y deberían ocupar hoy el lugar que les corresponde en el desarrollo de la larga historia andina.

Palabras clave: Arte andino, tejido andino, bordados Paracas, Estilo Lima Interlocking, práctica del tejido, diseños estructurales, formas geométricas.

\section{Le textile comme matrice du développement des arts plastiques dans les Andes RÉSUMÉ}

L'origine textile de nombreux dessins à tendance géométrique présents sur diverses catégories d'œuvres d'art a été abondamment commentée pour plusieurs cultures préhispaniques de la côte péruvienne, mais les rapports directs entre dessins et textiles n'ont jamais été étudiés avec attention. L'auteure démontre comment certaines pratiques de tissage actuelles des hautes terres andines permettent de lire ces dessins et de reconstruire les techniques dominante chaîne à l'origine de leur création. Pratiquées dans les hautes terres depuis au moins l'Horizon Ancien, ces techniques ne sont donc pas une invention récente malgré le nombre limité d'exemples conservés. Les nombreuses reproductions de leurs dessins à l'aide d'autres techniques textiles -broderie et tapisserie entre autres- ou à l'aide d'autres matériaux -peinture murale ou sur céramique, bois sculpté- réalisées sur la côte sud et la côte centrale du Pérou à la fin de l'Horizon Ancien et pendant l'Intermédiaire Récent fournissent un moyen inespéré de reconstruire leur passé décomposé et de retracer les relations entre côte et hautes terres à des périodes où elles ne sont pas faciles à détecter. Ces tissus dominante chaîne ont bien joué un rôle important dans l'histoire de l'art andin et ils devraient désormais occuper la place qui leur revient dans le développement de la longue histoire andine.

Mots clefs: Art andin, textiles andins, broderies Paracas, Style Lima Interlocking, pratique du tissage, dessins structurels, formes géométriques.

\section{Textile as Matrix for the Development of Visual Arts in the Andes}

\begin{abstract}
The observation that numerous geometric designs on artwork of various media from several pre-Hispanic cultures of the Peruvian coast appear similar to those found in textiles has been made in the past. The direct links between the designs and textiles, however, has never been examined in detail. As a means to identify the actual sources for this extensive design program, the author shows how the understanding of some present weaving practices from the Andean highlands can help in the decipherment of this relationship, and in particular in the reconstruction of the warp-faced techniques underlying their creation. In use in the highlands since at least
\end{abstract}


the Early Horizon, these techniques are not a recent invention, though we know only a few examples from the period. The numerous reproductions of these warp-patterned designs made with other media, such as seen on ceramic and metalwork from both the South Coast and the Central Coast of Peru at the end of the Early Horizon and during the Early Intermediate Period, provide new means to reveal and analyse the relationship not only between media, but between the cultures of the coast and the highlands at periods when they are not easy to detect, due to the lack of material and the subtle evidence of their interaction. These warp-faced textiles have played an important role in the history of Andean art and they should now take their rightful place in being recognized for their contribution to the development of Andean culture and history.

Key words: Andean art, Andean textiles, Paracas embroideries, Lima Interlocking style, weaving practices, structural designs, geometrical shapes.

Sumario: 1. Prácticas de tejido y diseños estructurales de los jalq'a de Bolivia. 2. Otros conteos con urdimbres complementarias y diseños geométricos más frecuentes. 3. Diseños estructurales creados con urdimbres complementarias durante la época prehispánica. 4. Conclusión. 5. Post scriptum. 6. Referencias bibliográficas.

«Es posible sostener, sin ser exagerados, que el tejido fue en el área andina la matriz primaria para el desarrollo de las artes plásticas. De su seno surgieron las escuelas y tendencias que se expresaron luego en la pintura mural, el grabado en metal, madera o concha, el modelado en barro o el diseño en cerámica y aún en la rígida escultura en piedra.

Por eso, el análisis del arte antiguo del Perú debe comenzar con el estudio del tejido si se quiere entender no solamente sus mecanismos de cambio al interior de cada cultura, sino también las fuentes de unidad estilística e ideológica a lo largo de éste tan variado como heterogéneo país de los Andes centrales», L. G. Lumbreras (1977: 8).

Es con estas palabras que Luis Guillermo Lumbreras justifica la selección del textil como tema del primer volumen de una colección de libros sobre el arte y el patrimonio peruano ${ }^{1}$. Para nosotros que vivimos en una sociedad que considera el arte textil como un «arte menor» o «decorativo», la desorientación es total. Sin embargo, debemos reconocer el nivel artístico excepcional de muchos textiles prehispánicos que nos han llegado gracias a las condiciones de conservación muy favorables del clima desértico de la costa. Debemos también recordarnos el valor central del textil en las sociedades andinas tal como los españoles lo observaron en el siglo XVI y aun después (Murra 1989), y tal se puede notar hoy en día en comunidades de las tierras altas.

Para demostrar, en el ámbito de los Andes Centrales, la influencia de los textiles sobre las otras artes plásticas, Lumbreras hace énfasis en varias de sus características, en particular la importancia de «diseños estructurales» generados, en el caso de los tejidos, al mismo tiempo que estos, mediante el entrecruzamiento de los hilos de urdimbre -tensos longitudinalmente en el telar- con los hilos de trama que son introducidos transversalmente entre los primeros. El incide en la tendencia geométrica de esos «diseños textiles» basados en el patrón rectilíneo de la urdimbre y de la trama, y los opone a los diseños sin limitaciones formales producidos en la superficie plana de un tejido ya existente con técnicas tales como el bordado o la pintura (Lumbreras 1977: 14-15) ${ }^{2}$. Luego observa la proporción importante de «diseños estructurales» en

${ }^{1}$ Esa colección fue creada y dirigida por José Antonio de Lavalle y Werner Lang y publicada por el Banco de Crédito del Perú a partir de fines de los años setenta.

2 Bird $(1961,1963 a)$ ya había insistido sobre el problema de los diseños estructurales, con más detalles. 
el arte textil andino, y destaca su importancia en obras de arte producidas con varios otros medios de expresión en diversas regiones y épocas. Un ejemplo muy característico de este fenómeno se encuentra en la costa central del Perú en el Intermedio Temprano ${ }^{3}$ con muchas cerámicas del estilo «Lima» llamadas «interlocking» por el carácter geométrico de sus diseños pintados, en particular de serpientes bicéfalas entrelazadas, que se consideran concebidos dentro del mundo textil.

A pesar de que estamos de acuerdo con esa interpretación de la influencia de diseños textiles de formas geométricas sobre el arte andino, sostenemos una posición un poco diferente de la de Lumbreras. Primero, todos los «diseños estructurales» no son geométricos - existen motivos bastante realistas tejidos por ejemplo con la técnica de tapiz-. Segundo, se puede llegar a varios grados de estilización según las técnicas usadas para crearlos. Tercero, dentro del mundo textil hubo ya mucha influencia de los diseños creados con las técnicas más «constrictivas» en oposición a los que fueron producidos con técnicas más «libres». De ello se derivan varias preguntas a las cuales propondremos unas respuestas: ¿Cuáles son las técnicas usadas por las tejedoras prehispánicas que producen los diseños más geométricos y por qué? ¿Se puede hacer una tipología de los diseños estructurales geométricos que permita entender cómo han podido circular dentro de la esfera textil? Y ¿cuáles son los diseños estructurales que han sido usados como modelos por artistas trabajando con otros medios?

Es cerca de las tejedoras actuales que hemos investigado para encontrar respuestas a nuestras preguntas ${ }^{4}$. En efecto, a pesar de que las prácticas textiles, todavía muy vivas en varias regiones de las tierras altas del Sur del Perú y de Bolivia, ofrecen a nuestra admiración tejidos que parecen muy alejados de las producciones prehispánicas -con sus diseños de caballos, letras y guitarras, por ejemplo-, las lógicas empleadas para su fabricación tienen mucho en común con las usadas en el pasado y especialmente en lo que concierne a la creación de diseños. La observación participante (llevada a cabo en 1979, 1983 y 2012) de las tejedoras jalq'a de las tierras altas del sur de Bolivia, así como de otras tejedoras bolivianas, nos ayudó a entender la diversidad de las prácticas de tejer y de los tipos de diseños estructurales obtenidos, y a especificar los principios que llevan a la creación de diseños que pueden ser más o menos geométricos. Empezaremos por lo tanto a analizar las prácticas de tejido actuales y los límites formales que imponen sobre los diseños. Luego examinaremos el caso de las técnicas más constrictivas y propondremos una tipología de los diseños obtenidos con ellas. Finalmente, volveremos a los tejidos prehispánicos para entender cómo esos principios han podido dar forma, en el pasado, a una serie de diseños textiles que han circulado dentro y fuera del mundo textil con materiales muy variados.

3 Como es la más usada para los textiles precolombinos, nos referiremos a la cronología relativa establecida por J. Rowe (1962).

4 Con la excepción de los casos en que los artistas del textil sean en su mayoría hombres (como los tejedores de tapiz inca, por ejemplo), hemos decidido no aplicar la regla gramatical que requiere, en caso de plural, el uso del masculino. Dado que las personas que tejen hoy en el telar andino, y en la zona considerada, tejidos cara de urdimbre son en su mayoría mujeres, usaremos el término femenino también para la época precolombina. 

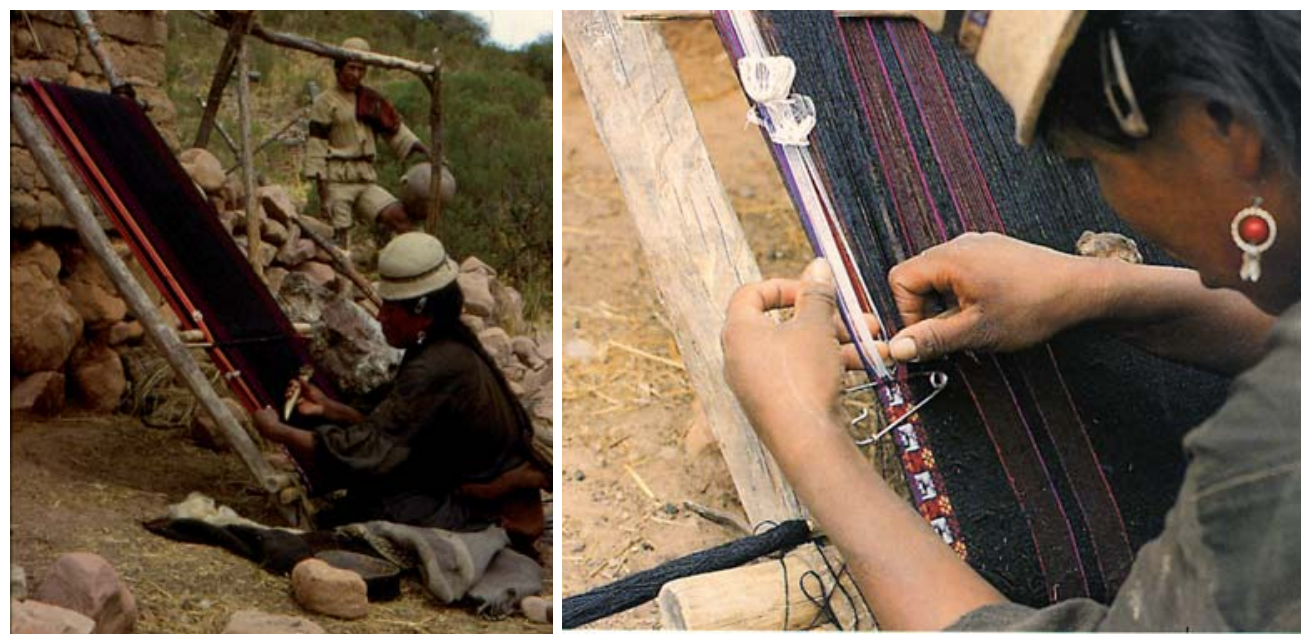

Figura 1: Telar jalq'a, Isluco, provincia de Chayanta, departamento de Potosí, Bolivia, 1983:

a) tejiendo la mitad de un poncho en el telar andino con estructuras cara de urdimbre; b) selección de los hilos de urdimbre en una banda con pallay en doble tejido con tres urdimbres. (C Desrosiers).

\section{Prácticas de tejido y diseños estructurales de los jalq’a de Bolivia}

Los jalq'a viven de la agricultura en valles y punas bajas, entre los 2.500 y 3.500 metros de altura, al oeste y al noroeste de la ciudad de Sucre, a caballo entre las provincias de Oropeza (departamento de Chuquisaca) y Chayanta (departamento de Potosí). No se sabe mucho del pasado de este grupo de habla quechua, tampoco de la antigüedad de su estilo textil que sería bastante reciente según las investigaciones de Verónica Cereceda ${ }^{5}$. Hoy en día, junto con sus mitos de origen y sus rituales, la vestimenta de los jalq'a juega un papel importante en la definición de su identidad (Cereceda et al. 1993).

Las tejedoras jalq'a tejen en un telar andino oblicuo (Figura 1). Conforme a la tradición prehispánica, sus tejidos tienen cuatro orillos y nunca son cortados al momento de hacer los vestidos cuyos nombres y formas son muy parecidos a los que llevaban los Incas: un aqsu (reducido a un sobre-vestido), un ch'umpi (faja) y una llijlla (chal) para las mujeres (Figura 2), un poncho que ha sustituido al unku (túnica cosida en ambos lados) y una ch'uspa, pequeña bolsa para llevar hojas de coca, para los hombres. Todas esas piezas están tejidas cara de urdimbre, es decir, con los hilos de urdimbre -tensos en el telar- tan densos que esconden los hilos de trama introducidos transversalmente entre ellos.

Incluidas en el tejido llano, se destacan bandas con diseños producidos por el contraste entre hilos de urdimbre urdidos juntos en el telar: uno rojo, anaranjado o

\footnotetext{
5 Según Verónica Cereceda (comunicación personal 2012), el tejido más antiguo que puede ser fechado no antecede a 1898, y fue regalado por la comunidad de Potolo a la niña de la hacienda que se casaba ese año. El actual territorio de los jalq'a estaba ocupado en parte por la población Yampara-que ocupaba el valle de Sucre a la llegada de los españoles en el siglo XVI- y por un segmento de los Moromoros que formaban parte del Señorío de los Caracaras, aliados de los Charcas (Cereceda et al. 1993: 8-10; Barragán 1994: 44).
} 

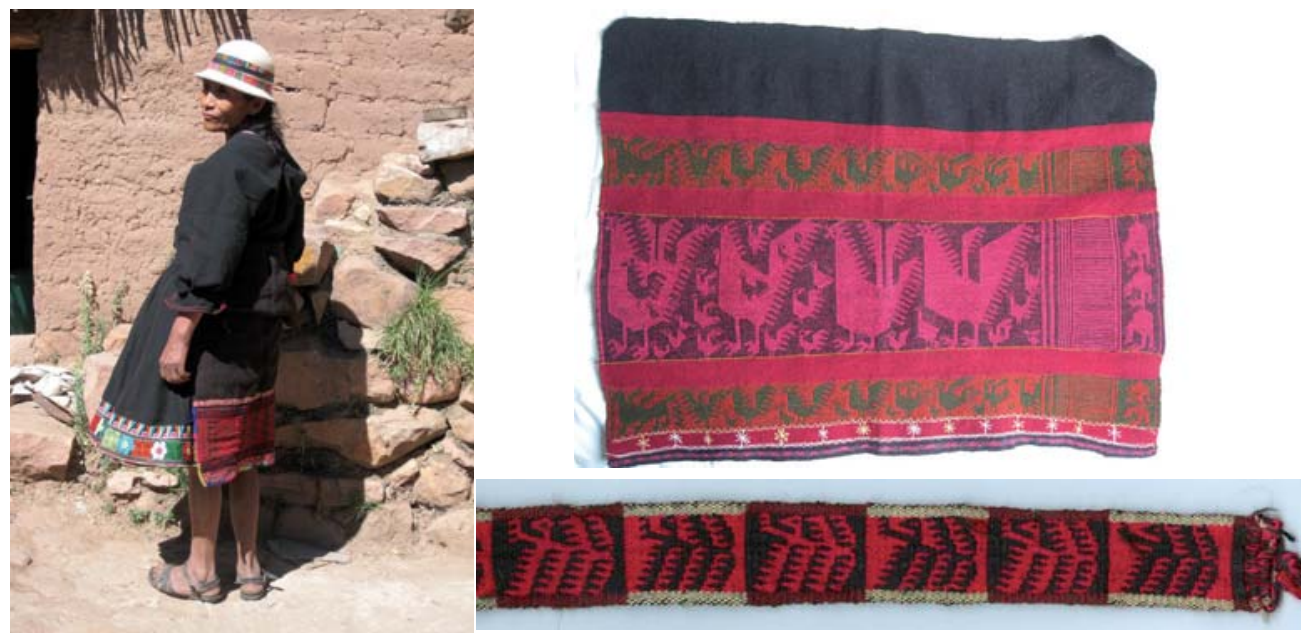

Figura 2: a) Encima de un vestido de corte europeo, las mujeres jalq'a llevan tres piezas de forma inca con diseños tejidos en el telar (pallay): el aqsu, amarrado en la cintura por un ch'umpi, es ahora de tamaño reducido y cubre solamente la parte de detrás del cuerpo (la mitad superior del aqsu no se ve porque está enrollada encima del ch'umpi); ese día de julio de 2012, en Isloco, Máxima Calle no llevaba su llijlla (chal); b) y c) medio aqsu y ch'umpi de los años 80. Los tejidos están aquí dispuestos con los hilos de urdimbre en horizontal. (C Desrosiers).

rosado, otro negro o a veces azul o verde oscuro. Esos diseños aparecen en las dos caras, pero con formas simétricas y colores invertidos. Puede ocurrir que una tercera urdimbre añada un color contrastante en los ch'umpis o en la banda situada al borde de tejidos de tamaño mayor (Figuralb) - pero el resultado tiene siempre sus dos caras con diseños de la misma calidad-.

Desde 1993 Verónica Cereceda $(1993,2010)$ ha descrito cómo los jalq'a señalan su identidad textil en los pallay ${ }^{6}$ de sus aqsus a través de una especie de caos poblado por seres fantásticos, los khurus no domesticados y unos seres entre dioses y demonios intercalados con ellos, los supay o saxra. A pesar de que nuestros ejemplos son piezas tejidas treinta años atrás con diseños menos caóticos que los aqsus más recientes, veremos que esa complejidad encuentra un eco en la manera de escoger los hilos para crear los diseños.

Para ellos, las tejedoras jalq'a utilizaban y todavía utilizan una estructura muy característica con dos urdimbres complementarias que es mucho más complicada de tejer que la otra estructura, el doble tejido, que usan para realizar tanto sus ch'umpis como bandas estrechas en otros varios tejidos. Por necesitar dos urdimbres y una trama, habitualmente esta estructura se considera más simple de tejer que el doble tejido, el cual necesita un número más importante de series de hilos (dos urdimbres y dos tramas para obtener dos tejidos sobrepuestos) $)^{7}$. No obstante, seleccionar los hilos para hacer un diseño en doble-tejido es mucho más fácil y el diseño encuentra menos limitaciones formales que el pallay con hilos complementarios (Desrosiers 2012a y b).

\footnotetext{
${ }^{6}$ La palabra quechua pallay designa un diseño tejido mediante la selección de hilos de diferentes colores.

7 Usaremos aquí la terminología descriptiva establecida por A. Rowe (1977) y ampliamente reconocida por los investigadores.
} 


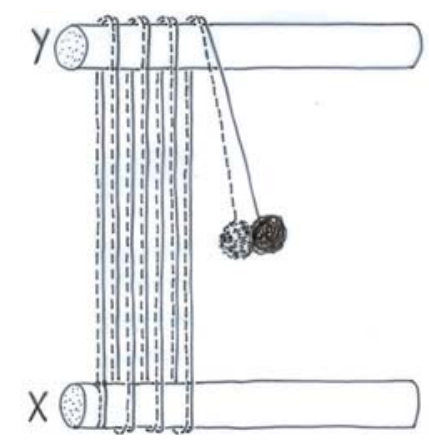

Figura 3: Urdido de los pares de hilos -uno rojo y el otro negroen forma de ocho entre las dos barras del urdidor (X e Y).

(C) Desrosiers).

Para demostrar esas diferencias, compararemos los procesos técnicos usados para tejer los dos grupos de tejidos centrándonos solamente en las tres etapas determinantes para nuestra demostración: el urdido, la construcción del dispositivo para controlar los hilos -lizos y separador-y la selección de los hilos necesaria para obtener los diseños.

Primera etapa: el urdido. En las secciones con pallay, las tejedoras jalq'a urden los hilos en el telar de la misma manera para las dos técnicas: con una pareja de hilos de urdimbre - generalmente uno negro y el otro rojo $^{8}$ - en un movimiento en forma de ocho entre las dos barras del urdidor (Figura 3). Estos dos hilos constituyen una pareja conceptual durante todo el proceso, pues si se selecciona un hilo de la pareja, el otro, que es de un color contrastante, tiene que aparecer atrás para construir un dibujo simétrico con colores opuestos. Es una lógica muy simple que funciona maravillosamente. Esas parejas de hilos constituyen una representación material del principio de complementariedad relacionado con el dualismo andino. Los términos descriptivos que usamos para las estructuras permiten pensar que ese principio está presente únicamente en las técnicas denominadas «con urdimbres complementarias». Pero la observación de la práctica demuestra que es indispensable también para obtener las dos caras del tejido doble 9 .

Segunda etapa: la construcción de los dispositivos para controlar los hilos difiere entre el tejido doble y las estructuras con urdimbres complementarias. En el primer caso, se conserva la distinción entre las parejas de hilos impares urdidas por encima de la barra inferior del urdidor, y las parejas de hilos pares urdidas por encima de la barra superior (Cuadro 1a). Los hilos impares están incluidos individualmente en los lizos y se introduce el separador para controlar los hilos pares (Cuadro 1b) ${ }^{10}$. En el caso de las urdimbres complementarias, se reorganizan los hilos pares y los impares

\footnotetext{
8 Nos quedaremos con la oposición negro/rojo en todas las explicaciones a pesar de que los ejemplos figurados tienen colores variables. Cada ida y vuelta - es decir dos hilos de cada color- es considerada como una unidad de urdido.

9 Ya en 1990, Doyon-Bernard (1990: 81) consideraba el tejido doble como basado también en la complementariedad de los hilos de urdimbre.

10 Ese dispositivo ha sido observado en 2012. En 1983, observamos otro: con el uso de dos lizos (1 y 2) para los hilos impares - uno para cada color-y un lizo (3) con el separador (4) para los hilos pares - de nuevo uno para cada color (ver Desrosiers 2012a)-. A pesar de esa diferencia en el dispositivo para controlar los hilos, no cambia mucho el procedimiento para escoger los hilos durante la tercera etapa.
} 
Cuadro 1: a) Cruce entre las parejas de hilos impares y las parejas de hilos pares tal como resulta del urdido; b) construcción de los dispositivos para controlar los hilos en 2012 para el tejido doble; c) lo mismo para el tejido con dos urdimbres complementarias.

\begin{tabular}{lll}
\hline \multirow{2}{*}{ a. } & $\mathrm{Y}$ (parejas de hilos pares): & $\mathrm{R}^{2} \mathrm{~N}^{2} \mathrm{R}^{4} \mathrm{~N}^{4} \mathrm{R}^{6} \mathrm{~N}^{6} \mathrm{R}^{8} \mathrm{~N}^{8}$ \\
& $\mathrm{X}$ (parejas de hilos impares): & $\mathrm{R}^{1} \mathrm{~N}^{1} \mathrm{R}^{3} \mathrm{~N}^{3} \mathrm{R}^{5} \mathrm{~N}^{5} \mathrm{R}^{7} \mathrm{~N}^{7}$ \\
\hline \multirow{2}{*}{ b. } & Separador: & $\mathrm{R}^{2} \mathrm{~N}^{2} \mathrm{R}^{4} \mathrm{~N}^{4} \mathrm{R}^{6} \mathrm{~N}^{6} \mathrm{R}^{8} \mathrm{~N}^{8}$ \\
& Lizo: & $\mathrm{R}^{1} \mathrm{~N}^{1} \mathrm{R}^{3} \mathrm{~N}^{3} \mathrm{R}^{5} \mathrm{~N}^{5} \mathrm{R}^{7} \mathrm{~N}^{7}$ \\
\hline \multirow{2}{*}{ c. } & Separador: & $\mathrm{N}^{1} \mathrm{~N}^{2} \mathrm{~N}^{3} \mathrm{~N}^{4} \mathrm{~N}^{5} \mathrm{~N}^{6} \mathrm{~N}^{7} \mathrm{~N}^{8}$ \\
& Lizo: & $\mathrm{R}^{1} \mathrm{R}^{2} \mathrm{R}^{3} \mathrm{R}^{4} \mathrm{R}^{5} \mathrm{R}^{6} \mathrm{R}^{7} \mathrm{R}^{8}$ \\
\hline
\end{tabular}

de tal manera que los hilos rojos se cruzan en el centro con los hilos negros, uno por uno. Luego se construyen los lizos para controlar los hilos rojos y se introduce el separador para los negros (Cuadro 1c).

Tercera etapa: las prácticas para seleccionar los hilos y obtener un diseño. Concentran las diferencias más significativas. Empieza después de haber finalizado la orilla trasversal en la barra definitiva del telar.

Con el tejido doble, cara de urdimbre (Figura 4a), la tejedora escoge tanto en las parejas impares controladas por el lizo para las líneas impares del diseño, como en las parejas pares controladas por el separador para las líneas pares (Figura 3). Empezando con las parejas impares, escoge un hilo de cada pareja para separar los que aparecerán en la cara superior, mientras que los hilos no seleccionados, de colores opuestos, aparecerán en la cara inferior (Figura 4b). Este procedimiento es muy ingenioso porque una sola selección basta para obtener las dos caras del tejido. Después de haber introducido la trama para la cara superior, la tejedora agrega a la selección todos los hilos pares controlados por el separador de tal manera que aparecen abajo solamente los hilos impares no seleccionados. Encima de ellos se puede introducir la trama para la cara inferior.

Como se ve en la Figura 4c y el Cuadro 2, los hilos aparecen como puntos independientes conforme al papel cuadriculado que hemos preparado para representar las selecciones de hilos. La tejedora selecciona los hilos de derecha a izquierda, de abajo hacia arriba.

Para una línea par del diseño, la tejedora continúa con el mismo procedimiento a partir de las parejas pares controladas por el separador: escoge un hilo de cada pareja, introduce la trama superior, agrega los hilos impares a la selección para introducir la trama inferior (Figura 4b). Es bastante simple. La práctica conceptual consiste en «dibujar» un animal línea por línea, escogiendo en cada pareja el color que conviene para obtener el diseño. Es como si se leyera el papel cuadriculado sobre el cual se ha pintado el diseño de derecha a izquierda y de abajo hacia arriba, una línea después de la otra, cada una independiente de la precedente. La tejedora debe saber cómo formar el animal de esta manera particular. En concreto, en la línea 3 (Figura 4c) debe saber dónde escoger el primer hilo rojo y el segundo para figurar las puntas de las orejas del caballo. Y en la línea 5 debe saber dónde empezar la cola para que el caballo tenga un cuerpo equilibrado. No tiene otra limitación que el número de hilos disponibles, el cual equivale al número de hilos urdidos: cuantos más hilos, más detalles posibles, como los píxeles en la pantalla de una televisión. En el caso de los tejidos jalq'a, las fajas y los otros tejidos en doble tejido, tienen diseños muy parecidos a los creados con urdimbres complementarias, como vamos a ver, pero esto no se debe a limita- 
Cuadro 2: Número de hilos negros $(\mathrm{N})$ y rojos $(\mathrm{R})$ escogidos para las primeras tramas al componer el caballo de la Figura $4 \mathrm{c}$ (el cuadro se debe leer de derecha a izquierda y de abajo arriba, conforme a la práctica de tejer).

\begin{aligned} \hline 4N 1R 1N 1R 3N 1R 1N 1R 12N 1R 1N 2R 3N 2R 2N : separador $= & (6) \\ 5 \mathrm{~N} 1 \mathrm{R} 4 \mathrm{~N} 1 \mathrm{R} 15 \mathrm{~N} 2 \mathrm{R} 4 \mathrm{~N} 2 \mathrm{R} 2 \mathrm{~N}:$ lizo $= & (5) \\$ Todos los hilos negros que siguen... 2R 5N 2R 1N : separador $= & (4) \\$ Todos los hilos negros que siguen... 1R 6N 1R 2N : lizo $= & (3) \\$ Todos los hilos negros del separador $= & (2) \\$ Todos los hilos negros del lizo & $=(1) \\ & \end{aligned}$
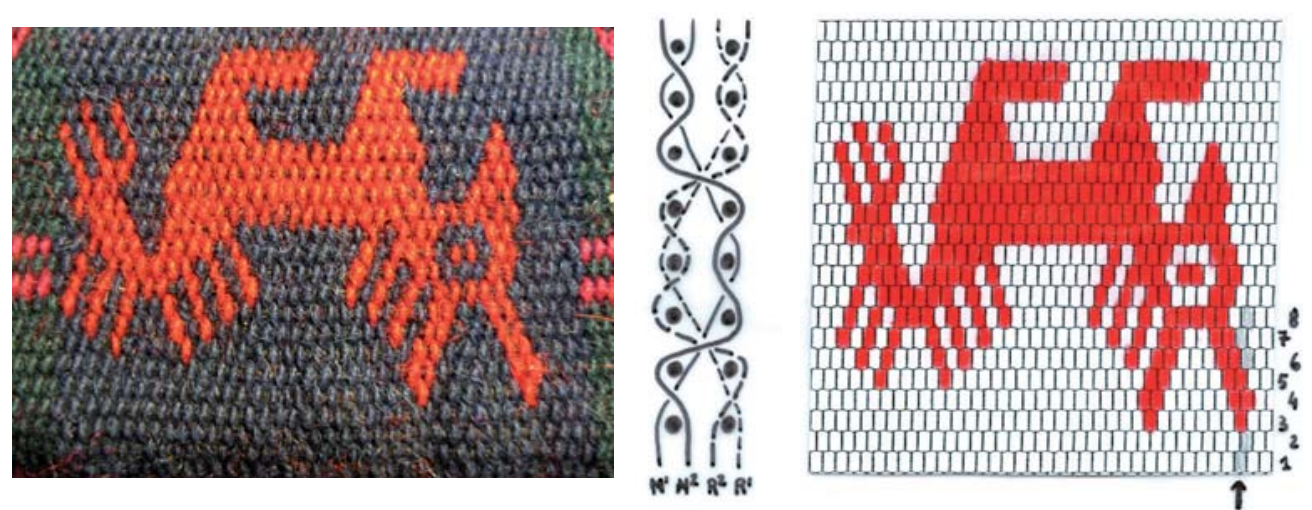

Figura 4: Doble-tejido en 2012: a) detalle del tejido de la Figura 6a; b) perfil del tejido doble con las urdimbres que cambian de cara; (c) representación de los hilos escogidos en papel cuadriculado. (C) Desrosiers).

ciones impuestas por la técnica, sino a la voluntad de la tejedora de usar motivos del repertorio considerado como característico de los jalq'a.

En el caso del tejido con urdimbres complementarias (Figura 5a), la tejedora trabaja el cruce entre los hilos rojos (controlados por el lizo) y los hilos negros (controlados por el separador) (véase la Figura 3). Selecciona siempre un solo hilo de cada pareja de hilos urdidos juntos. Pero, como las estructuras con hilos complementarios tienen por base hilos flotantes $-\mathrm{y}$ no puntos individuales como el tejido doble- la realización de estos requiere conteos rítmicos para realizar la estructura, además de la selección de los colores para crear el diseño. Examinaremos primero el caso de hilos flotantes de 2/1 organizados en líneas paralelas oblicuas -el sistema preferido por los jalq'a - y luego el caso de hilos flotantes de $3 / 1$ organizados en parejas alternadas que se encuentra mezclado con el precedente en un grupo de tejidos probablemente de una zona particular del territorio jalq'a.

\subsection{Urdimbres complementarias con hilos flotantes de 2/1 organizados en líneas paralelas oblicuas}

Como se ve en la Figura 5 y en el Cuadro 3, la tejedora tiene que contar los hilos con ritmos diferentes en las zonas de cada color, de derecha a izquierda, de abajo hacia 
Cuadro 3: Número de hilos negros $(\mathrm{N})$ y rojos $(\mathrm{R})$ escogidos para las primeras tramas de la Figura 5c. Los términos subrayados señalan dónde se producen cambios de colores

(lectura de derecha a izquierda y de abajo arriba).

$\begin{array}{r}\hline \\ 2 \mathrm{~N} 1 \mathrm{R} 2 \mathrm{~N} 1 \mathrm{R} 2 \mathrm{~N} 1 \mathrm{R} 2 \mathrm{~N} 1 \mathrm{R} 2 \mathrm{~N} 1 \mathrm{R} 2 \mathrm{~N} 2 \mathrm{R} 1 \mathrm{~N} 2 \mathrm{R} 1 \mathrm{~N} 2 \mathrm{R} 1 \mathrm{~N} 2 \mathrm{R} 1 \mathrm{~N}=(6) \\ 1 \mathrm{R} 2 \mathrm{~N} 1 \mathrm{R} 2 \mathrm{~N} 1 \mathrm{R} 2 \mathrm{~N} 1 \mathrm{R} 2 \mathrm{~N} 1 \mathrm{R} 2 \mathrm{~N} 1 \mathrm{R} 2 \mathrm{~N} 2 \mathrm{R} 1 \mathrm{~N} 2 \mathrm{R} 1 \mathrm{~N} 2 \mathrm{R} 1 \mathrm{~N} 2 \mathrm{R}=(5) \\ 1 \mathrm{~N} 1 \mathrm{R} 2 \mathrm{~N} 1 \mathrm{R} 2 \mathrm{~N} 1 \mathrm{R} 11 \mathrm{~N} 2 \mathrm{R} 1 \mathrm{~N} 2 \mathrm{R} 1 \mathrm{~N} 2 \mathrm{R} 1 \mathrm{~N} 1 \mathrm{R}=(4) \\ 2 \mathrm{~N} 1 \mathrm{R} 2 \mathrm{~N} 1 \mathrm{R} 2 \mathrm{~N} 1 \mathrm{R} 1 \mathrm{~N} 2 \mathrm{R} 1 \mathrm{~N} 2 \mathrm{R} 1 \mathrm{~N} 2 \mathrm{R} 1 \mathrm{~N}=(3) \\ 1 \mathrm{R} 2 \mathrm{~N} 1 \mathrm{R} 2 \mathrm{~N} 1 \mathrm{R} 2 \mathrm{~N} 2 \mathrm{R} 1 \mathrm{~N} 2 \mathrm{R} 1 \mathrm{~N} 2 \mathrm{R} 1 \mathrm{~N} 2 \mathrm{R} 1 \mathrm{~N} 2 \mathrm{R} 1 \mathrm{~N} 2 \mathrm{R} 1 \mathrm{~N} 2 \mathrm{R}=(2) \\ 1 \mathrm{~N} 1 \mathrm{R} 2 \mathrm{~N} 1 \mathrm{R} 2 \mathrm{~N} 1 \mathrm{R} 2 \mathrm{~N} 2 \mathrm{R} 1 \mathrm{~N} 2 \mathrm{R} 1 \mathrm{~N} 2 \mathrm{R} 1 \mathrm{~N} 2 \mathrm{R} 1 \mathrm{~N} 2 \mathrm{R} 1 \mathrm{~N} 2 \mathrm{R} 1 \mathrm{~N} 1 \mathrm{R}=(1) \\ \hline\end{array}$
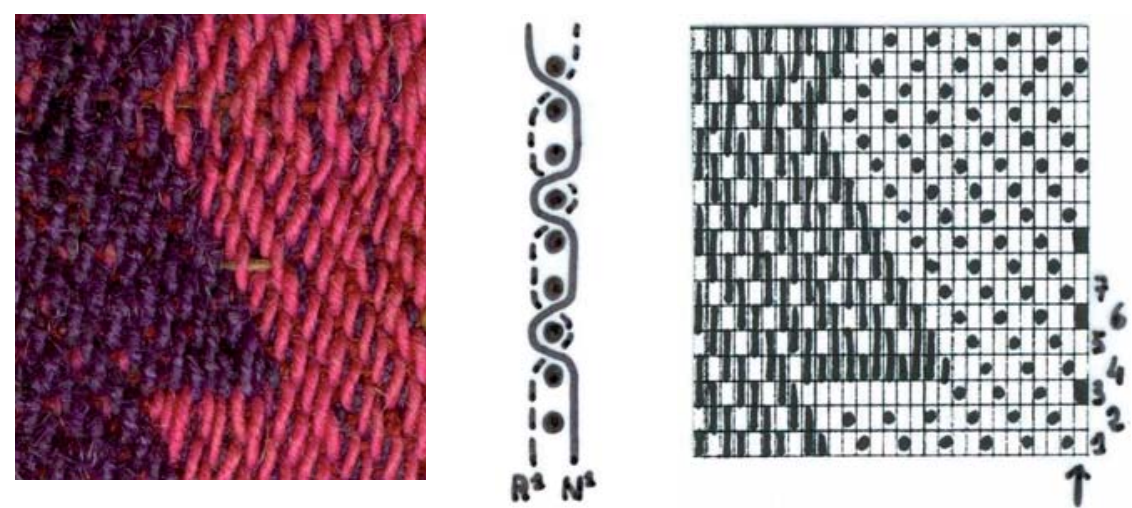

Figura 5: Tejido con dos urdimbres complementarias e hilos flotantes de 2.1 en líneas oblicuas: a) detalle del tejido; b) perfil del tejido con las urdimbres opuestas; c) representación de los hilos flotantes en papel cuadriculado. (C) Desrosiers).

arriba. Ella sigue el ritmo: 2 Rojos-1 Negro, 2R-1N, 2R-1N.... Cuando cambia de color, se para después de $2 \mathrm{R}$, y empieza la siguiente serie con los colores invertidos: 2N-1R, 2N-1R, 2N-1R... Para cada trama, el conteo empezará en un punto diferente del ritmo: por ejemplo $1 \mathrm{R}-1 \mathrm{~N}, 2 \mathrm{R}-1 \mathrm{~N}, 2 \mathrm{R}-1 \mathrm{~N} \ldots$ para la trama (1), seguido por $2 \mathrm{R}-$ $1 \mathrm{~N}, 2 \mathrm{R}-1 \mathrm{~N}, 2 \mathrm{R}-1 \mathrm{~N} \ldots$ para la trama (2), de tal manera que los hilos flotantes pasan por encima de dos tramas y se ordenan en líneas oblicuas en la dirección S.

Esta selección no sería tan complicada si las líneas oblicuas de los hilos flotantes fueran siempre en la misma dirección y si los cambios de colores siguieran siempre esas líneas. Pero el sentido de las líneas puede cambiar hacia la dirección $\mathrm{Z}$ o varias líneas pueden estar interrumpidas por un cambio de color horizontal, según la textura y el diseño deseados por la tejedora. Por ejemplo, en las tramas (3) y (4) donde el color cambia horizontalmente, la tejedora interrumpe el conteo regular con $\underline{11 \mathrm{R}}$, luego para la siguiente trama con $\underline{11 \mathrm{~N}}$. Ese número «11» responde a una fórmula matemática -tres veces el ritmo basado en 3 hilos, más los 2 necesarios antes de cambiar de colorcomo muchos otros cambios del ritmo básico de los hilos flotantes. Y como los tejidos jalq'a se caracterizan por sus diseños caóticos, escoger los hilos requiere calcular todo el tiempo los equilibrios necesarios para llegar al diseño deseado, con una restricción en la mente: los hilos flotantes no deben superar 3 tramas o, excepcionalmente, 4 tramas, de manera que el tejido no tenga largos hilos flotantes, los cuales introducen 

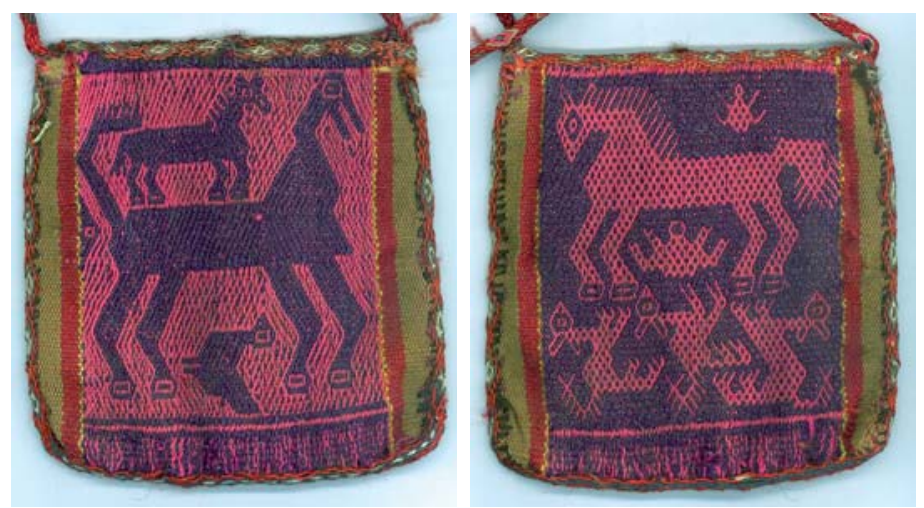

Figura 6: Como lo demuestran las dos caras de esta ch'uspa, algunas tejedoras jalq'a usan dos tipos de organización de los hilos flotantes: a) hilos flotantes de 2.1 en líneas oblicuas (ver Figura 5); b) hilos flotantes de 3.1 en pares alternados que resulta en una cara caracterizada por «marcadores» o puntos regularmente distribuidos. (C) Desrosiers).

debilidad física en el tejido al mismo tiempo que son antiestéticos. Eso se traduce en una regla muy útil para la tejedora, porque al controlar continuamente que los hilos que escoge no flotan ya sobre las 3 o 4 tramas precedentes, tiene puntos de referencia que le permiten escoger una parte de los hilos sin tener que contarlos uno por uno.

Sin embargo, la lógica del tejido con urdimbres complementarias es compleja y requiere mucha más capacidad de abstracción por parte de la tejedora que el doble tejido, porque necesita hacer una síntesis entre los principios de complementariedad y unos conteos y equilibrios matemáticos. Estructuralmente, cada introducción de la trama está relacionada con las precedentes. Gráficamente, la forma del diseño depende de las tres maneras de cambiar de color: a lo largo de dos líneas oblicuas de hilos flotantes orientadas en $\mathrm{Z}$ o en $\mathrm{S}^{11}$, y de líneas horizontales (Figuras 2b, 5, 6a). El tejido obtenido tiene una superficie caracterizada por líneas oblicuas que cambian de dirección en cualquier momento, y diseños de formas bastante geométricas, con detalles reducidos a triángulos, diamantes y hexágonos y, en el caso de plumas, pelos y algunas otras partes del cuerpo, a unidades simétricas repetitivas muy características. A pesar de esas dificultades y gracias a los puntos de referencia proporcionados por las selecciones precedentes, las tejedoras jalq'a consiguen tejer con bastante velocidad, como un músico que conoce su partitura. Veremos ahora hasta qué punto la selección es diferente cuando cambia la organización de los hilos flotantes.

\subsection{Urdimbres complementarias con hilos flotantes de 3/1 organizados en parejas alternadas $^{12}$}

Como se ve en las Figuras $6 \mathrm{~b}$ y $7 \mathrm{a}$, el ritmo seguido por la tejedora para seleccionar los hilos es muy diferente. Contando los hilos siempre de derecha a izquierda, una

11 Esas líneas oblicuas tienen siempre el mismo ángulo agudo con la vertical porque este depende de la proporción entre la densidad de los hilos en la urdimbre y en la trama. En un tejido con urdimbres complementarias y con hilos del mismo diámetro, la proporción se aproxima a menudo a 1,8/2. Por eso, el papel cuadriculado usado para analizar los diseños tiene pequeños rectángulos cuyo largo es dos veces el ancho, lo que produce líneas oblicuas con ángulos aproximadamente de $30^{\circ}$ con el eje vertical.

12 No hemos podido observar tejedoras jalq'a tejiendo así. Las explicaciones que siguen vienen de la observación de los tejidos, de la comprensión de una lógica común y de la confirmación a través de la obser- 
Cuadro 4: Número de hilos negros $(\mathrm{N})$ y rojos (R) escogidos para las primeras tramas del diseño de la vizcacha de la Figura 7a (lectura de derecha a izquierda y de abajo arriba).

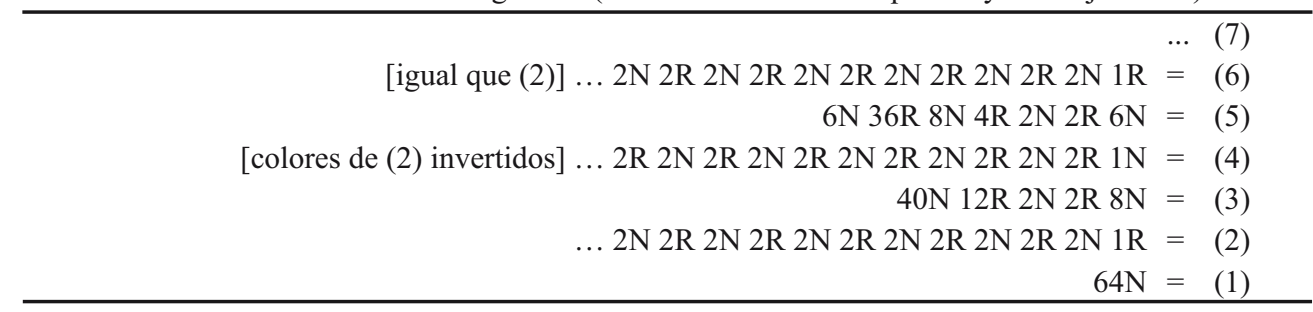
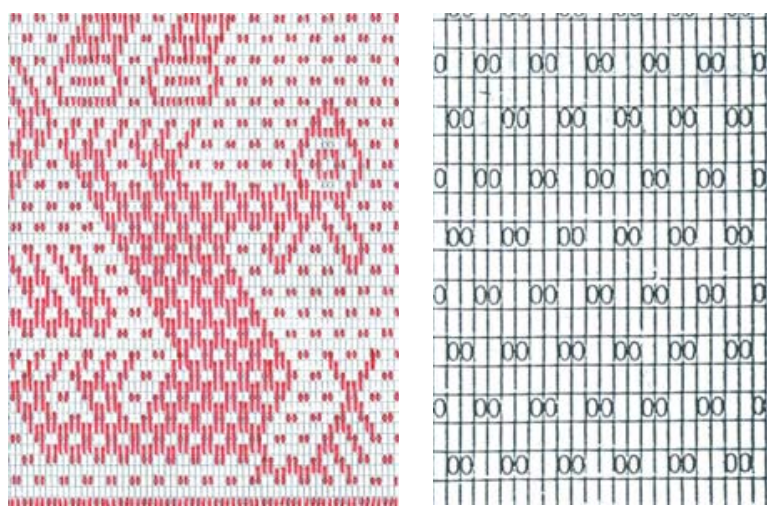

Figura 7: a) Representación en papel cuadriculado de los hilos flotantes formando el diseño de la vizcacha de la Figura $6 \mathrm{~b}(\mathrm{C})$ Desrosiers); b) papel cuadriculado con marcadores usado por Cason y Cahlander (1976).

línea después de la otra, desde abajo hacia arriba, sigue el código en el Cuadro 4 para realizar el diseño de la vizcacha (Lagidium viscacia, un roedor de la familia de la chinchilla).

La tejedora sigue dos ritmos diferentes. Para las líneas pares sigue un ritmo de 2 hilos de cada color: alternativamente empieza por $1 \mathrm{R}$ y sigue con $2 \mathrm{~N}-2 \mathrm{R}-2 \mathrm{~N}-2 \mathrm{R}$..., o empieza con $1 \mathrm{~N}$ y sigue con $2 \mathrm{R}-2 \mathrm{~N}-2 \mathrm{R}-2 \mathrm{~N}$... produciendo así los marcadores o puntos dobles de colores opuestos que se encuentran regularmente dispuestos en las áreas de cada color, en las dos caras del tejido. Para las líneas impares, selecciona números pares de hilos que corresponden al color de las diferentes zonas: $64 \mathrm{~N}$ para la línea 1 que es toda negra, luego $8 \mathrm{~N}, 2 \mathrm{R}, 2 \mathrm{~N}, 12 \mathrm{R}, 40 \mathrm{~N}$ para empezar la cola de la vizcacha en la línea 3 , y continúa con $6 \mathrm{~N}, 2 \mathrm{R}, 2 \mathrm{~N}, 4 \mathrm{R}, 8 \mathrm{~N}, 36 \mathrm{R}, 6 \mathrm{~N}$ para la línea 5 cuando aparece el cuerpo del animal. Así la selección es mucho más regular que en el caso precedente, no solamente por las líneas pares de marcadores, sino también por las impares, donde todos los números de hilos son pares. Se dan algunas excepciones cuando hay cambios de color horizontales: para la cara de la vizcacha o, arriba, para las pezuñas del caballo.

Después de constatar esa regularidad y de llamar a esa estructura «peeble weave» (estructura con guijarros), Cason y Cahlander (1976) han propuesto usar un papel especial, con los marcadores preparados (Figura 7b). Es una invención muy astuta porque ayuda a entender cómo está construido el diseño: basta relacionar los mar-

vación en 1979 de tejedoras en la zona de Llallagua (provincia Bustillo, departamento de Potosí). 
cadores con líneas oblicuas y unas pocas líneas horizontales (Figura 7a) ${ }^{13}$. Ayuda también a contar los hilos de cada color que se deben escoger para las líneas impares, porque cada pequeño trazo oblicuo es equivalente a un par de hilos. Por ejemplo, la línea 3 está compuesta de 4 trazos invisibles (equivalente a hilos negros no dibujados para no recargar el esquema), o sea, 4 pares de hilos negros $(8 \mathrm{~N}), 1$ trazo rojo $(2 \mathrm{R}), 1$ ausente $(2 \mathrm{~N}), 6$ rojos (12R), 20 ausentes $(40 \mathrm{~N})$ y la línea 5 de 3 ausentes $(6 \mathrm{~N}), 1$ rojo (2R), 1 ausente (2N), 2 rojos (4R), 4 ausentes $(8 \mathrm{~N}), 18$ rojos (36R) y 3 ausentes $(6 \mathrm{~N})$.

Resulta una comprensión muy clara, tanto del mecanismo conceptual como de las formas posibles de los diseños. Las tejedoras seleccionan los hilos con estos equilibrios en la mente. Después de haber escogido una línea con marcadores, muy regular, saben que los hilos de las líneas intermedias deben encontrarse a caballo entre esos marcadores y los siguientes y, para una línea horizontal, rompen temporalmente el ritmo de una línea. Esos equilibrios matemáticos son más simples que los precedentes porque son muy regulares y balanceados. Por eso dejan una marca muy fuerte en los diseños que tienen no solamente formas delimitadas por las mismas dos líneas oblicuas posibles y líneas horizontales, sino también marcadores distribuidos en cada área colorida a la misma distancia los unos de los otros y, por supuesto, también de los contornos de los diseños. Los pequeños elementos de la composición toman muy fácilmente formas de triángulo, diamante y hexágono, como aquí la cabeza de la vizcacha y las pezuñas del caballo (Figura 6b) o constituyen pequeñas series de líneas oblicuas paralelas, como ocurre en la cola y en las patas de la vizcacha, y en la cola y la melena del caballo.

De estas observaciones se deduce que los diseños de ambas estructuras con urdimbres complementarias encuentran las mismas limitaciones formales y por ello pueden llegar a formas bastante parecidas. Pero las texturas obtenidas son muy diferentes. Cuando se comparan los caballos tejidos con los dos ritmos (Figura 6a y b), parece que la presencia de los marcadores conduce a la creación de motivos con proporciones más equilibradas. Es probable que el conteo más característico de los tejidos jalq'a (2/1) deje más flexibilidad a la tejedora, por ejemplo, en el ancho variable de las patas y del cuello y esa «libertad» ayude en la creación de los seres fantásticos que caracterizan la identidad textil jalq'a. La red mental en la cual se apoyan las tejedoras es mucho más compleja conceptualmente y menos rígida, y favorece la elaboración de dibujos relativamente menos estandarizados.

\subsection{Tejidos con tres urdimbres de colores diferentes}

Antes de concluir esta primera parte, examinaremos brevemente los cambios introducidos cuando las tejedoras jalq'a tejen con tres urdimbres de colores diferentes el tejido doble y estructuras con urdimbres complementarias. Sin entrar en una descripción detallada de la práctica, se puede observar que el urdido es muy parecido al de la Figura 4a: siempre realizando un movimiento en forma de ocho entre las dos barras del urdidor, pero con tres hilos juntos - uno negro, uno rojo y un tercero aquí azul-. Pero

13 Según Ed Franquemont (1983: 48), las tejedoras de Chinchero los llaman ñawi-«ojo» en quechua-y el vocablo peeble era ya usado en Norteamérica por Birrell (1973: 140-143). En otro articulo (Franquemont 1987: 77), insiste sobre el hecho de que «speed and efficiency in Chinchero weaving come more from mental agility than from mechanical skill». 


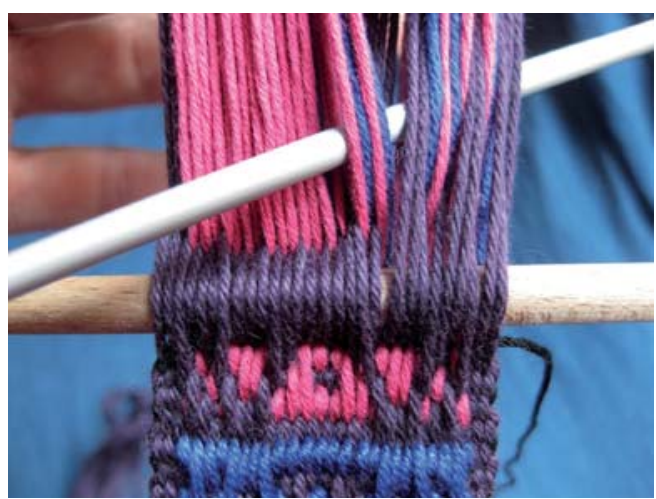

Figura 8: Pallay con 3 urdimbres: a) selección de los hilos para la trama 5 del diseño con rosado: los hilos de color están escogidos juntos como un elemento complementario de los hilos oscuros y son separados antes de introducir la trama; b) y c) las dos caras con colores opuestos después de separar los hilos e introducir una trama para cada cara; d) representación de los hilos flotantes negros en b y c en papel cuadriculado; e) perfil del tejido con los hilos rojos y azules opuestos a los hilos negros. (C Desrosiers).
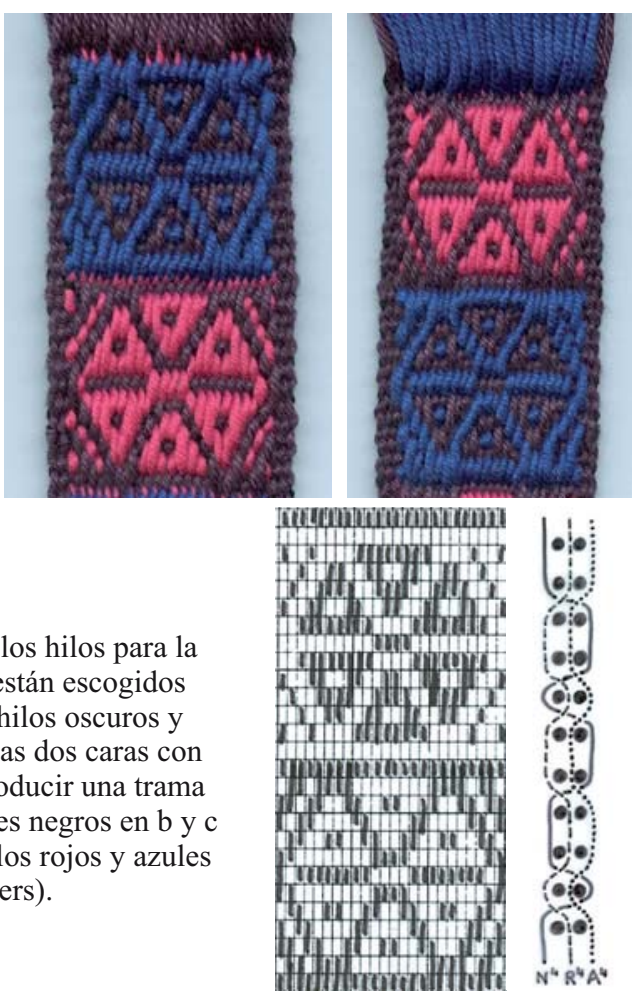

del proceso que sigue y del producto final, se entiende que los tres hilos no tienen el mismo rol porque un color (generalmente el más oscuro) se opone a los otros dos que se escogen juntos (Figura 8a). Luego esos dos hilos son separados -lo que las tejedoras llaman «re-escogido» según Arnold y Espejo (2012: 21 y 229-242) - de tal manera que el negro aparece en las dos caras (Figura 8c-e): en una cara, hace el diseño rojo sobre un fondo negro mientras que, en la otra cara, el diseño aparece negro sobre un fondo azul ${ }^{14}$. Se llega así a una forma de dualismo asimétrico correspondiente a la lógica de tripartición observada por Olivia Harris (1978) con los laymi y por Platt (1980) con los macha de Bolivia. Al igual que con dos urdimbres, la construcción con tres urdimbres del tejido doble y del tejido con urdimbres complementarias constituye una representación material del principio de complementariedad relacionado con el dualismo andino. Desde el punto de vista de la selección de los hilos y de los diseños obtenidos, no hay gran diferencia con el tejido con dos urdimbres. Funcionalmente, la introducción de una tercera urdimbre aumenta el número de colores disponibles pero no modifica la apariencia de las caras de los dos tipos de estructuras, ni el tipo de conteo necesario para crear el diseño con urdimbres complementarias ${ }^{15}$.

\footnotetext{
14 Como en el caso del tejido doble con dos urdimbres, la otra cara se obtiene gracias al uso de una segunda trama que pasa debajo de los hilos escogidos a los cuales las tejedoras juntan mecánicamente hilos controlados por un lizo o un separador.

15 A pesar de que la presencia de una tercera, o más, urdimbres cambia normalmente el término usado para denominar la estructura -ya que hay una lógica de sustitución que opera en el «re-escogido»-continuaremos
} 


\subsection{Conclusiones}

Como conclusión de esta primera parte sobre las prácticas conceptuales de las tejedoras jalq'a, podemos afirmar que hay una gran diferencia entre la creación de un diseño en tejido doble, basado en un ligamento llano cara de urdimbre, hecho por la yuxtaposición de puntos de colores diferentes, y la construcción de un diseño con urdimbres complementarias basado en hilos flotantes que están organizados con conteos rítmicos y equilibrios matemáticos. El primero puede llegar a formas bastante libres, según el proyecto y la habilidad de la tejedora, así como el número de hilos disponibles, mientras que el segundo produce diseños de formas geométricas, con contornos horizontales y oblicuos siempre con el mismo ángulo agudo con la vertical y detalles en forma de triángulos, diamantes, hexágonos y finas líneas oblicuas paralelas. Desde el punto de vista de la práctica conceptual, el carácter geométrico aparece relacionado con la complejidad matemática involucrada en el conteo de los hilos necesario para organizar los hilos flotantes que constituyen la superficie del tejido. En el caso de un tejido doble con ligamento llano cara de urdimbre, el eventual carácter geométrico del diseño depende de la elección de tal diseño por la tejedora o de su dificultad para liberarse de la dirección ortogonal de los hilos de urdimbre y de trama.

Como lo hemos explicado ya en otro trabajo (Desrosiers 2012b), se confirma que tejer con urdimbres complementarias es más complejo que tejer tejido doble y se evidencia que los dos tipos de técnica construyen sus dos caras simétricas gracias a la misma lógica de la complementariedad de los dos o tres hilos urdidos juntos, aunque solamente una manifiesta este hecho en su nombre. Por tanto, cuando se cambia de sistema referencial, en particular cuando se pasa de la sistematización de las estructuras al contexto de las prácticas de tejer, uno debe ser consciente de que las ideas y las palabras tienen que ser usadas con mucho cuidado.

Si comparamos ahora los dos tipos de organización de los hilos flotantes, vemos que cada uno tiene sus particularidades en la práctica. El tipo más característico del tejido jalq'a ordena los hilos flotantes según líneas oblicuas que pueden cambiar de dirección en cualquier momento, llamando así continuamente la atención de la tejedora. Sin embargo, el otro tipo se construye con normas fijas que, a pesar de ser mentales, se pueden representar en una cuadrícula que alterna tramas muy regulares, para las cuales se escogen secuencias de dos hilos de cada color, con tramas donde se decide la forma del diseño, escogiendo números pares de hilos de cada color. Desde el punto de vista del diseño, las diferencias más evidentes se concentran en la textura de las caras, en el equilibrio de las formas y en detalles como las plumas y los pelos más ondulados de los animales con el primer tipo. Vemos también que lo que visualmente aparece como «hilos flotantes de $2 / 1$ organizados en líneas oblicuas» se teje contando los hilos por $2 / 1$, y lo que aparece como «hilos flotantes de $3 / 1$ organizados por pares alternantes» ${ }^{16}$ se teje contando los hilos por $2 / 2$. Así llegamos a la

usando la expresión «con urdimbres complementarias» para esas variaciones.

16 Esa terminología descriptiva enfocada en la organización de los hilos flotantes ha sido detallada por Ann Rowe para las estructuras con urdimbres complementarias (1977: 67-80). En 1976, Cason y Cahlander habían identificado esas mismas variantes y les habían dado nombres relacionados con su aspecto visual (pebble weave, intermesh...). 
nomenclatura usada en quechua y aymara por las tejedoras actuales de Bolivia, una nomenclatura relacionada con las prácticas de tejer que ha sido registrada de manera muy convincente por Denise Arnold y Elvira Espejo (2012). Por lo tanto, se entiende aquí también la diferencia de visión entre la descripción del objeto y la práctica para crearlo.

\section{Otros conteos con urdimbres complementarias y diseños geométricos más frecuentes}

El sistema aymara/quechua registrado por Arnold y Espejo (2012) es muy lógico. En lo que se refiere al tejido con urdimbres complementarias, demuestra que las tejedoras investigadas llaman a sus técnicas por los conteos rítmicos que usan para crear sus diseños: $1 / 1,2 / 1,2 / 2,3 / 3$ y $4 / 4{ }^{17}$. No parece que exista hoy una tradición textil local que maneje todos esos conteos y sería interesante saber si algunas manejan uno solo. Por lo que hemos podido observar con los jalq'a, las tejedoras tejen con el conteo 2/1 sus diseños más característicos, los que construyen su identidad textil, y algunas de ellas asocian el conteo $2 / 2$ en varias maneras - por ejemplo en la mitad de una ch'uspa (Figura 6)-. En muchas ocasiones observadas en 1983, tejían también bandas estrechas con otros conteos (Figura 8), pero con diseños repetitivos que no necesitaban una creatividad particular.

Para conectar la nomenclatura utilizada por las tejedoras bolivianas con la terminología descriptiva de Rowe, muy utilizada hasta ahora, presentamos los términos en paralelo en el Cuadro $5^{18}$.

En el cuadro están representados dos tipos de estructuras. Unas tejidas con conteos regulares -1/1, 2/2, 3/3, y 4/4- producen hilos flotantes alternados-simples, por pares, por tres y por cuatro- (Figura $9 \mathrm{a}, \mathrm{b}$ y d). Otra que se teje con conteo binario $-2 / 1$ - produce hilos flotantes en «diagonal alignment» que hemos analizado ya en detalle (Figuras 5, 6a). El Cuadro 5 indica además que, en dos casos de conteos regulares, $2 / 2$ y $4 / 4$, se producen también hilos flotantes en alineamiento diagonal: «2.2 twill» $\mathrm{y}$ 《2.2 twill with floats in pairs» (una sarga cuadrada 2.2 «simple» $\mathrm{y}$ «con hilos flotantes en pares») (Figura 9c y d). Los diseños que resultan son muy diferentes y llegan a definir dos estilos de diseños que se pueden producir con los conteos $2 / 2 \mathrm{y}$ 4/4: diseños de «estilo con áreas de color», donde los colores están divididos en bloques distintos (Figura 9b y d), y diseños de «estilo lineal», que presentan un diseño construido únicamente con líneas oblicuas y horizontales (Figura 9c y e).

\footnotetext{
17 A estos se pueden agregar conteos encontrados en tejidos publicados o examinados personalmente: $3 / 1$, 4/2, 6/2 y 6/3 y unos conteos híbridos por ser diferentes según las zonas del diseño. Se llega así a casi todas las estructuras posibles dentro de un marco limitado por el largo máximo de los hilos flotantes que, por razones de solidez del tejido, no debe sobrepasar tres tramas, a menos que el tejido sea muy fino. Se encuentran excepcionalmente hilos flotantes sobre 4 tramas en zonas con diseños complejos, en particular en los tejidos jalq'a con conteo $2 / 1$.

18 Nos limitamos aquí a los casos en que los hilos de urdimbre se siguen en el orden $a b a b$ como lo practican las tejedoras jalq'a. Los otros órdenes como abbabaab son relacionados por las tejedoras con otro grupo de tejido llamado peinecillo según Arnold y Espejo (Cason y Cahlander 1976: 83-88; A. Rowe 1977: 68-69; Franquemont 1983; Arnold y Espejo 2012: 155-166).
} 
Cuadro 5: Equivalencia entre los dos sistemas clasificatorios con 2 urdimbres y con hilos ordenados $a b a b$.

\begin{tabular}{lll}
\hline \multicolumn{1}{c}{$\begin{array}{c}\text { Manera de contar los hilos en quechua y } \\
\text { aymara (Arnold y Espejo 2012: 203-226) }\end{array}$} & Figuras & $\begin{array}{c}\text { Terminología descriptiva de } \\
\text { Ann Rowe (1977: 67-80) }\end{array}$ \\
\hline $\begin{array}{l}\text { Conteo por impar básico, 1/1 } \\
\text { maya palla (aymara), uj pallay (quechua) }\end{array}$ & $9 \mathrm{a}$ & 3-span floats in alternating alignment \\
$\begin{array}{l}\text { Conteo por impar derivado, 2/1 } \\
\text { ch'ulla palla (aymara), iskay uj pallay (quechua) }\end{array}$ & $5,6 \mathrm{a}$ & 2-span floats in diagonal alignment \\
$\begin{array}{l}\text { Conteo por par, 2/2 } \\
\text { parís palla (aymara), iskay pallay (quechua) }\end{array}$ & 6b, 9b & $\begin{array}{l}\text { 3-span floats alternating in pairs } \\
\text { Conteo por tres, 3/3 }\end{array}$ \\
$\begin{array}{l}\text { kimsa palla (aymara), kinsa pallay (quechua) } \\
\text { Conteo por cuatro, 4/4 }\end{array}$ & & 3-span floats alternating in threes* \\
pusi palla (aymara), tawa pallay (quechua) & $9 \mathrm{~d}$ & 3-span floats alternating in fours \\
\hline
\end{tabular}

* Nombre reconstruido por analogía con los demás nombres.

Para explicar de manera simple esa conexión, hemos representado en papel cuadriculado los conteos por par, 2/2, necesarios para obtener los diseños de la Figura $9 \mathrm{~b}$ y $9 \mathrm{c}$ (Figura 10a y b). Se ve claramente que corresponden a dos maneras distintas de contar los hilos para las tramas intermedias o tramas sin marcadores. En el caso del «estilo con áreas de color», el color de los grupos de pares de hilos escogidos para esas tramas determina el color de la zona y cada zona tiene marcadores del color opuesto. En el caso del «estilo lineal», se escogen los hilos por par, 2/2, como para las tramas con marcadores -es así que se obtiene al final una sarga 2.2 (lo escribo de manera diferente porque es una sarga y no un conteo)-, menos cuando el diseño sigue líneas horizontales. Finalmente, las tejedoras usan un tercero estilo, intermedio, que podemos llamar «estilo semi-lineal», donde se escogen pares de hilos de vez en cuando para formar líneas oblicuas y horizontales entre áreas de color (Figura 10c). Resulta un diseño de líneas menos densas, con marcadores dispuestos a una distancia muy regular de ellas. En los tres casos, las formas de los diseños son muy geométricas y tan características que se pueden reconocer sobre cualquier material ${ }^{19}$.

Los tres estilos se encuentran también con conteos por cuatro, $4 / 4$, pero en este caso las líneas oblicuas son escalonadas y menos agudas; la Figura 9c y 9e ilustra diseños representando dos de ellos. El conteo por impar básico, 1/1, no entra en la presente discusión porque su base «1» elimina la posibilidad de obtener formas geométricas como lo enseña la cabeza de caballo de la Figura 9a. En cierto modo, la flexibilidad relativa que permite se puede comparar al tejido doble que hemos examinado en la primera parte. En cuanto al conteo por tres, 3/3, su uso muy limitado, probablemente por el manejo difícil de tres hilos para construir diseños, no permite hacer observaciones sobre las formas de los diseños creados con él.

Los tres estilos que acabamos de definir están a menudo tejidos con más de dos urdimbres -hasta ocho urdimbres hoy en día por ciertas tejedoras de Bolivia (Arnold y Espejo 2012: 223-240)-. En el caso de los estilos «lineal» y «semi-lineal», los colores

19 Para esos estilos no hemos buscado una terminología que pueda conectarse con la que usan las tejedoras para los conteos porque no hemos investigado esa cuestión, y no sabemos si ellas tienen términos para hacer esas diferencias. 
Figura 9: Detalles de tejidos bolivianos con diseños creados por conteos regulares a) $1 / 1$; b) y c) $2 / 2$; d) y e) $4 / 4$. (El conteo $3 / 3$ no está representado por la escasez de ejemplos). Los diseños de a, b y d, contrastando zonas de colores diferentes, se consideran de «estilo con áreas de color». Los de c y e, hechos solamente de líneas, se consideran de «estilo lineal». (C) Desrosiers).
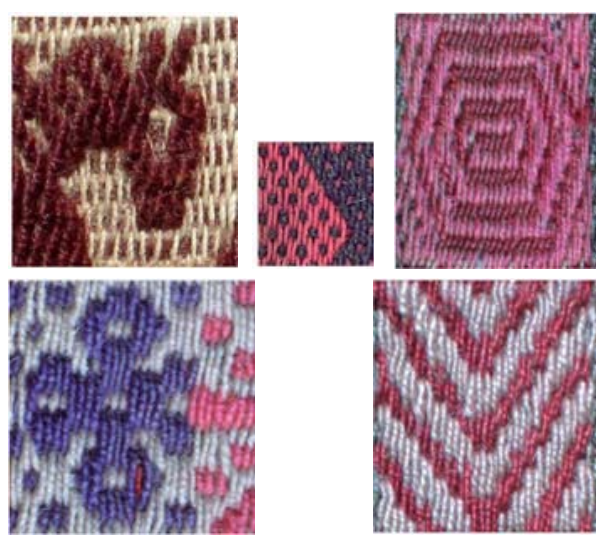
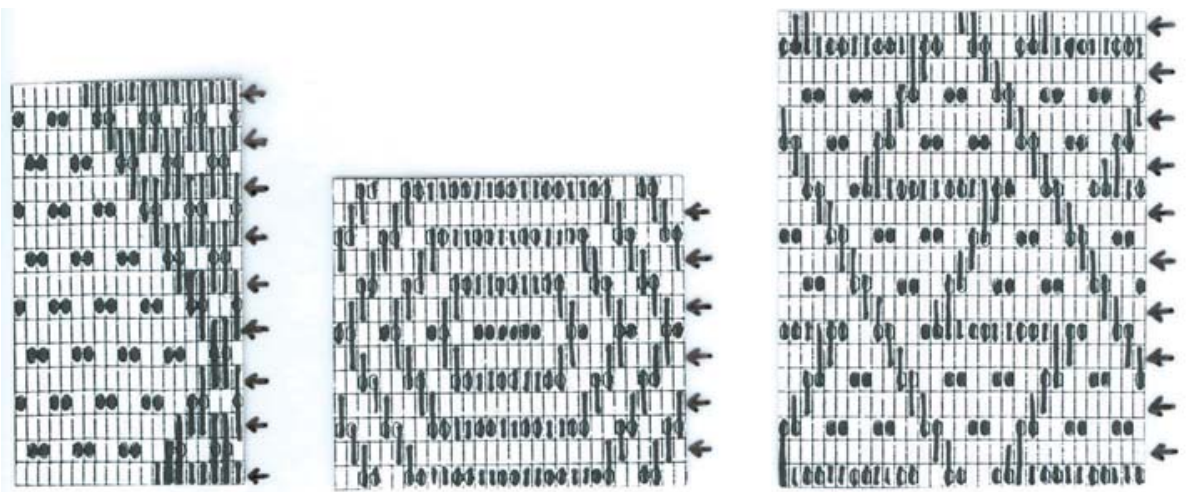

Figura 10: Representación en papel cuadriculado de los tres estilos de diseños que se pueden tejer con el conteo por par, 2/2. Cada estilo esta determinado por la manera de escoger los hilos en las tramas sin marcadores (marcadas por flechas): a) «estilo con áreas de color» de la Figura 9b obtenido al escoger grupos de pares de hilos que determinan el color de la zona (C Desrosiers); b) «estilo lineal» de la Figura 9c obtenido al escoger los hilos por par, 2/2, menos cuando el diseño sigue líneas horizontales (C) Desrosiers); c) «estilo semi-lineal» de un tejido de Chumbivilcas (Callañaupa 2007: 79) obtenido al escoger pares aislados de hilos en las tramas sin marcadores.

llenan las formas delimitadas por las líneas de tal manera que estas se convierten en los contornos de las figuras representadas. Según Arnold y Espejo (2012: 23, 229-242), las tejedoras llaman ese procedimiento «re-escogido» y distinguen dos tipos: «por unidad» cuando se refiere a una figura (Figura 8), y «por cantidad» cuando se refiere a una zona delimitada conteniendo varias figuras (ver más adelante Figuras 14c y 16).

No volveremos aquí a los diseños creados con conteo por impar derivado, 2/1, que no tienen otro estilo que «con áreas de color», al menos en los tejidos actuales y a pesar de que se pueden encontrar detalles con cambios de colores seguidos semejantes al «estilo lineal».

Antes de concluir esta parte, haremos una última observación sobre los diseños creados con conteos por par, 2/2. Hasta ahora hemos ilustrado un solo caso con los marcadores alineados en las mismas tramas cualquiera que sea el color-como lo 


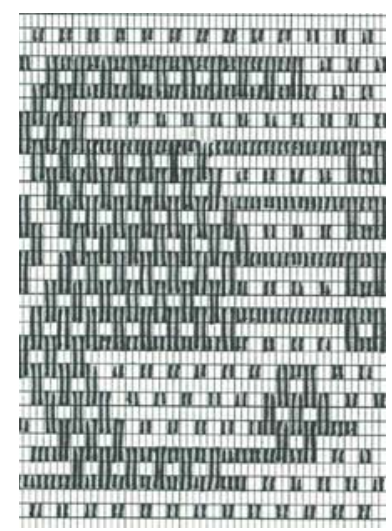

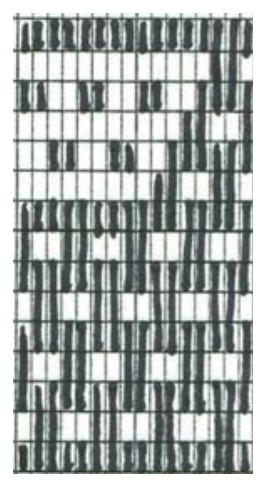

Figura 11: Diseños de «estilo con áreas de color» tejidos con conteo por par, 2/2: a) variante con marcadores en líneas desfasadas y forma escalonada (detalle de una ch'uspa de Pitumarka,

Perú [Rowe 1977: fig. 53]); b) variante con marcadores en líneas desfasadas y forma suave (detalle de un tejido de Chinchero, Perú [E. Franquemont 1983: fig. 2]).

enseñan los ejemplos jalq'a-, una variante que llamaremos «alineada» (Figura 7a). Para entender lo que sigue, debemos añadir dos variantes que tienen sus tramas con marcadores desfasados, es decir, que los marcadores se encuentran en una trama para un color y en la trama siguiente para el otro color (Figura 11). En un caso, las formas son escalonadas y las llamaremos variante «desfasada-escalonada». En la otra, donde las formas son suaves (en el sentido de no escalonadas), las consideremos simplemente variante «desfasada». Nos limitaremos aquí a identificarlos. Explicaremos las posibles razones técnicas e históricas de esas variaciones en otro lugar.

Finalmente, antes de examinar a partir de cuándo y cómo los diseños identificados han circulado dentro y fuera del mundo textil en la época prehispánica, haremos dos observaciones sobre prácticas bastante recientes.

La primera concierne a una bufanda en ligamento cara de trama tejida en un telar de pedal por el hombre que la llevaba y que estaba viajando un día de 1979 entre la ciudad de Sucre y su comunidad al norte del departamento de Potosí (Figura 12a). Intercaladas entre zonas en ligamento llano cara de trama, tenía barras horizontales con diseños creados con dos tramas complementarias cambiando de color en la altura de cada barra. Esas tramas formaban hilos flotantes 3/1 en pares alternados y, en consecuencia, diseños con marcadores que eran la traducción cara de trama de un pallay creado en el telar andino con dos urdimbres complementarias y un conteo por par, $2 / 2$. El hombre habría imitado en el telar de pedal unas bandas originalmente tejidas cara de urdimbre por las tejedoras de su comunidad, girándolas $90^{\circ}$. Esa manera de tejer implica lógicas y equilibrios matemáticos más simples que los usados para tejer el original cara de urdimbre ${ }^{20}$. Y si la comparamos con la técnica de tapiz que, desde hace algunos años, es practicada por hombres jalq'a de ASUR ${ }^{21}$ (Figura 12b), nos damos cuenta de que son dos maneras diferentes de crear diseños. Para simplificar, se puede decir que la creación de un diseño con la técnica de tapiz -o ligamen-

\footnotetext{
20 Recientemente nos hemos dado cuenta que O’Neale (1942: 156) pensaba lo mismo: «Warp-patterns of any sort usually indicates more foresight, preparation, and skill than do weft-made patterns».

21 ASUR, Antropólogos del Surandino: Fundación de Investigaciones Antropológicas con programa de etno-desarrollo destinado a dar un nuevo impulso a la producción textil de comunidades indígenas del centrosur de Bolivia. Fue creada por V. Cereceda y G. Martínez a partir de 1985.
} 
Figura 12: Dos técnicas que producen diseños cara de trama: a) bufanda con barras tejidas con dos tramas complementarias produciendo diseños de «estilo con áreas de color» con marcadores que son la traducción cara de trama de un pallay creado en el telar andino con dos urdimbres complementarias y un conteo por par, $2 / 2 ; b)$ tejedor jalq'a de ASUR tejiendo un panel en técnica de tapiz o tejido llano cara de trama con tramas discontinuas. (C) Desrosiers).
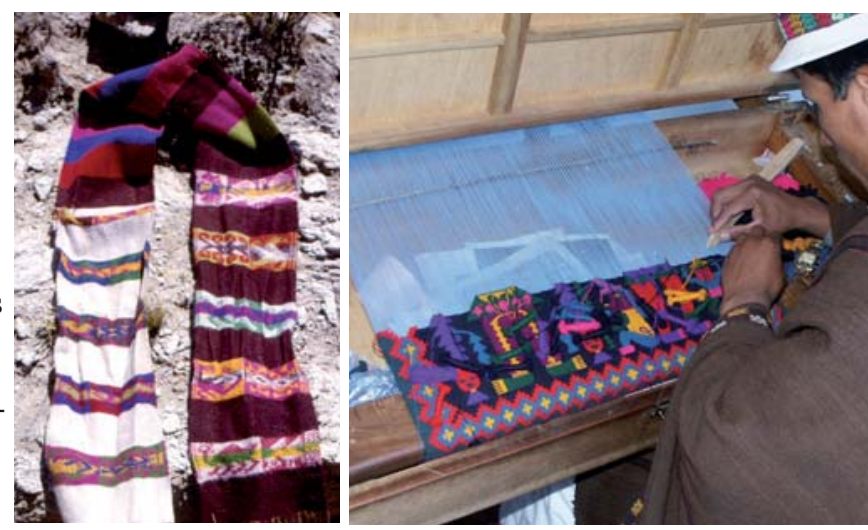

to llano cara de trama con tramas discontinuas- es equivalente a dar colores a los puntos-píxeles de una pantalla de televisión, como el tejido doble cara de urdimbre que hemos examinado más arriba, pero con un número de colores no limitado por la técnica ${ }^{22}$. Esta práctica requiere mucha habilidad y experiencia para colocar los colores en los puntos-píxeles necesarios para obtener curvas que están liberadas de las direcciones ortogonales de la urdimbre y de la trama, pero no exige el entendimiento de los números y de sus relaciones indispensable para tejer diseños, por ejemplo, de animales, con tramas complementarias. Desde el punto de vista de la calidad de los tejidos, la técnica de tapiz produce una textura muy llana como el tejido doble, mientras que la práctica de tejer con urdimbres o tramas complementarias da una textura que parece importante para las tejedoras que lo comentan.

La segunda observación concierne a la reproducción de diseños textiles con otros materiales. En el verano de 2012, durante una corta estancia en la isla de Taquile, hemos fotografiado el pavimento del restaurante comunal que representa los mismos diseños que las famosas fajas «calendario» llevadas por hombres y mujeres de una manera muy vistosa (Figura 13). Y es solamente escribiendo este artículo que nos hemos dado cuenta de que ilustraba una manera contemporánea de considerar el diseño textil como modelo para otras expresiones plásticas. Entender cómo fue decidido ese detalle de la obra de construcción del restaurante en el año 2007, dos años después de que los saberes textiles de la isla de Taquile fueran elegidos Patrimonio Oral e Inmaterial de la Humanidad por la UNESCO, está al alcance de una próxima estancia. Mientras tanto, ayuda a entender cómo diseños textiles muy presentes en un cierto contexto pueden volverse parte del vocabulario emblemático de una comunidad ${ }^{23}$.

\footnotetext{
22 Como lo hemos visto arriba, el número de colores del tejido doble cara de urdimbre está limitado por el número de urdimbres en el telar.

23 Muchos otros ejemplos se podrían identificar hoy en día en la manera de usar esos diseños en la publicidad, en particular por parte de las agencias de turismo locales e internacionales.
} 


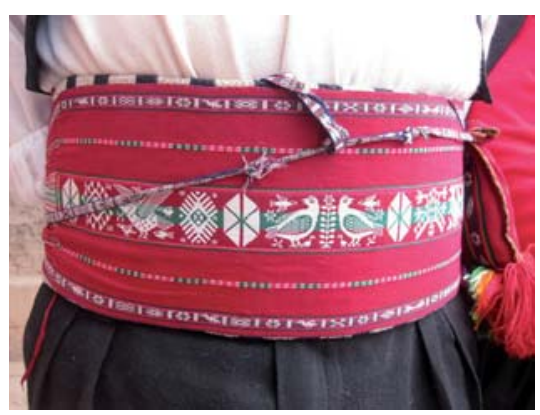

Figura 13: Isla de Taquile en el lago Titicaca, departamento de Puno, Perú: a) faja «calendario» con la banda central tejida en tejido doble cara de urdimbre; b) pavimento del restaurante comunal que representa, con guijarros blancos sobre guijarros rosados con raya negra en el centro, unos diseños tejidos en las fajas «calendario». Se debe notar la conexión formal y de color entre los guijarros y los puntos-pixeles que forman los diseños de la faja. (C Desrosiers).

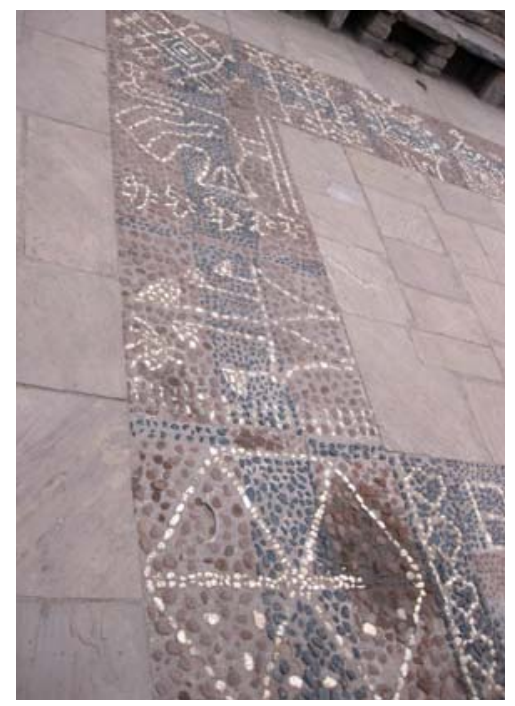

\section{Diseños estructurales creados con urdimbres complementarias durante la época prehispánica}

Siguiendo la terminología usada por las tejedoras bolivianas (Arnold y Espejo 2012) y la tipología de los diseños que acabamos de presentar, nos preguntaremos ahora desde cuándo y en qué forma los diseños textiles aquí caracterizados han podido desarrollarse y encontrarse fuera de la esfera de los tejidos con urdimbres complementarias. Examinaremos primero los más antiguos testimonios de esos tejidos que hemos podido identificar hasta la fecha a finales del Horizonte Temprano (fase 9-10, ca. 300-100 a.C.) en la Costa Sur del Perú, y trataremos de su influencia sobre técnicas y diseños locales. Nos interesaremos luego por las varias maneras en que han podido servir de modelos a unos grupos de bordados encontrados en Paracas Necrópolis ( $c$ a 150 a.C-200 d.C.). Finalmente, trataremos el caso muy especial de los diseños del estilo «interlocking» de la cultura Lima, en la Costa Central, en el Periodo Intermedio Temprano (ca. 200-650 d.C.).

\subsection{Antiguos testimonios de tejidos con urdimbres complementarias y su influencia sobre las prácticas textiles en la Costa Sur, fases 9 y 10 del Horizonte Tempra- no (ca. 300-100 a.C.)}

En dos artículos anteriores (Desrosiers 2008 y 2012a), hemos ya insistido sobre la complejidad de las técnicas con urdimbres complementarias que se encuentran con dos, tres y cuatro urdimbres, en tres bandas tejidas de fibra de camélido, sin contexto arqueológico, de estilo Ocucaje 9 y 10 (Figura 14) ${ }^{24}$.

24 Esas tres bandas fueron publicadas por King (1965: 174-176) y A. Rowe (1977: fig. 76, 98, 99). Wallace (1979: 44 y fig. 8) menciona un tejido con urdimbres complementarias muy temprano en Cerrillos (fase 3 de la secuencia de Ocucaje, $c a$. 800-650 a.C.). Sin embargo, se refiere a la terminología de Emery (1980). A. Rowe 

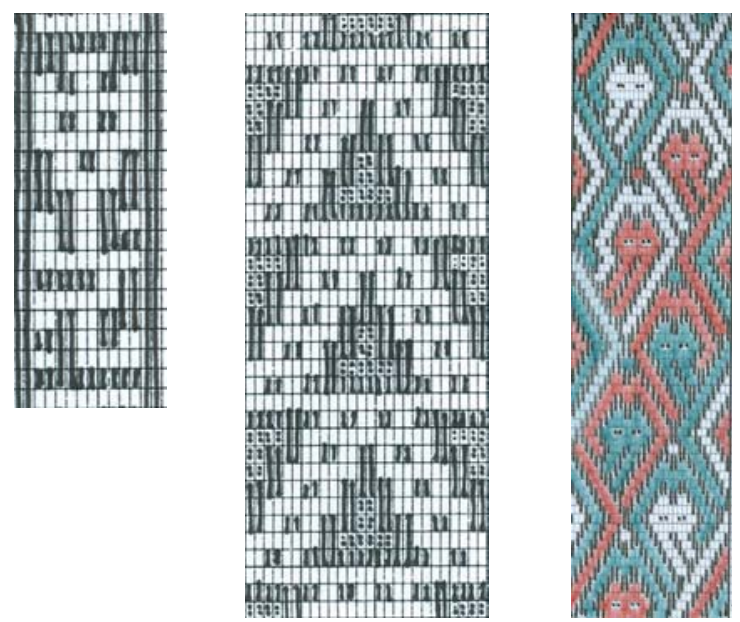

Figura 14: Diseños de las tres bandas con urdimbres complementarias conservadas en el TMW, de estilo Ocucaje 9 y 10 (Desrosiers 2008: fig. 3a, 8; 2012a: fig. 13):

a) 2 urdimbres, conteo por par, $2 / 2$ (parte superior), «estilo con áreas de color», variante desfasada-escalonada;

b) con 3 urdimbres, conteo por par, 2/2, «estilo semi-lineal», variante escalonada, «re-escogido por unidad»;

c) con 4 urdimbres, conteo por par,

2/2, «estilo lineal», con «re-escogido por cantidad».

La más simple, con dos urdimbres - una morada y la otra marrón verdoso o amari1lo-, tiene un diseño de un ser que parece correr. El diseño está compuesto en la parte superior por hilos flotantes sobre 3 tramas (a veces sobre 5) organizados en pares alternados. Fue parcialmente creado con el conteo por par $2 / 2$, que se identifica en la cabeza. La parte baja del cuerpo tiene demasiado movimiento como para seguir un conteo regular. A pesar de su tamaño reducido, se puede considerar como afiliado al «estilo con áreas de color», en su variante «desfasada-escalonada».

La banda siguiente es más ancha y tiene tres urdimbres ${ }^{25}$. Su diseño de triángulos concatenados con el interior y los bordes verdes, y el resto blanco, tiene contornos y marcadores negros. Contrariamente a los ejemplares etnográficos, tiene una sola cara; la otra se puede considerar como un revés donde los hilos temporalmente inútiles producen largos hilos flotantes. El diseño está compuesto por hilos flotantes sobre 3 tramas organizados en pares alternados, menos en los contornos, donde esos pares se siguen en líneas oblicuas escalonadas. Fue creado con el conteo por par $2 / 2$. Tiene características del «estilo semi-lineal», en su variante «escalonada» y los hilos han sido «re-escogidos por unidad».

La última banda es aún más ancha. Su diseño de serpientes entrelazadas tiene contornos negros; los otros colores -rojo, blanco y verde- se intercambian para presentar serpientes en bandas oblicuas sucesivamente verdes sobre rojo, rojos sobre blanco, y blancos sobre verde. Tiene también una sola cara. El diseño está compuesto por hilos flotantes sobre 2 tramas organizados en líneas oblicuas. No hay marcadores sino los ojos de las serpientes. Fue creado con el conteo por par $2 / 2$ y es de «estilo lineal» con hilos «re-escogidos por cantidad».

Por varias razones, incluyendo la alta complejidad de las bandas con tres y cuatro colores, la diversidad de los estilos y de sus variantes, y el hecho de que son los

(1977: 50-52) lo hubiera considerado como tejido con una urdimbre de sustitución. En 1990, Doyon-Bernard (1990: 78-79) había llegado a la misma conclusión.

25 Según A. Rowe (1977: 81), la urdimbre verde está presente solamente en el centro y en los bordes, donde es necesaria para llenar las formas cerradas por las líneas oscuras. 
más antiguos ejemplares con urdimbres complementarias que hemos podido localizar hasta ahora, parece claro que fueron producidos por tejedoras de una región donde los tejidos no se han conservado y no pueden entonces atestiguar el desarrollo progresivo de esa técnica ${ }^{26}$. Las tierras altas, donde esas prácticas están todavía muy vivas, son el mejor candidato para localizar ese desarrollo, en una época bien anterior a la fase 9 de Ocucaje. Además, las diferencias de gamas de colores y estilos de diseños aliadas a las de técnicas, sugieren que cada banda, o al menos las dos primeras de un lado y la tercera del otro, han sido tejidas por tejedoras con referencias culturales diferentes, quizás en áreas diferentes.

Al observar los otros tejidos de estilo Ocucaje estudiados por M. E. King (1965), y otros tejidos contemporáneos encontrados en la región, se puede notar que la presencia de esos tejidos en la Costa Sur ha influenciado las prácticas locales de varias maneras.

Primero, parece haberse producido allá el mismo fenómeno observado en 1979 en la bufanda del norte de Potosí, es decir: la imitación de un tejido con urdimbres complementarias girado $90^{\circ}$ para tejerlo con tramas complementarias (Figura 15). Esa estructura con tramas de fibra de camélido ocupa los dos extremos de un tejido llano de algodón encontrado en 2003 en Cerrillos, en el valle alto de Ica, considerado de la fase 9 de Ocucaje ${ }^{27}$. El diseño se caracteriza por triángulos concatenados. Su modelo hipotético cara de urdimbre habría sido creado con el conteo por par, 2/2, en el «estilo semi-lineal» escalonado. Es muy interesante constatar que tiene, no solamente las mismas características que la segunda banda de Ocucaje girada $90^{\circ}$ y con una urdimbre menos, sino que su diseño también es a base de triángulos (Figura 14b).

Esa semejanza estructural e iconográfica parece explicable por el deseo de imitar los ejemplos cara de urdimbre desarrollados en las tierras altas. Es bastante común en la historia del tejido y de otras producciones humanas que un objeto, al llegar a una área con prácticas conceptuales diferentes de las usadas en la región de su fabricación -en este caso tejidos decorados por la trama ${ }^{28}$-, es recreado con una técnica más conforme a los instrumentos y saberes locales ${ }^{29}$. Y es así que entendemos la presencia de esa técnica en Cerrillos: como una imitación no solamente del diseño, sino también de la técnica, en una zona que privilegiaba otras técnicas de decoración textil.

Segundo, un fenómeno similar de imitación de la estructura y del diseño parece presente en varias bandas con diseños de «estilo lineal»o «semi-lineal» (los últimos más o menos simplificados porque los marcadores no han sido siempre reproducidos). Los diseños de esas bandas bastante estrechas fueron esta vez producidos por hilos de urdimbre, en piezas de estilo Ocucaje y en otras contemporáneas descubier-

\footnotetext{
26 Los famosos tejidos de Huaca Prieta no están considerados aquí por no estar tejidos con el principio de complementariedad de las urdimbres (Bird 1963b; Bird et al. 1985: 194-197).

27 Este tejido envolvía un textil muy particular definido como «Spiral-Wrapped Batons and Cords» por Jeffrey Splitstoser cuando lo presentó en el simposio Textiles, Techne and Power in the Andes en Londres, 1517 Marzo 2012 (ver Splitstoser en prensa).

28 Dos técnicas de creación de diseños por las tramas: el tapiz y las tramas suplementarias (o bordado en tejido llano que los imita) eran bastante usadas en la Costa Sur en el Horizonte Temprano, ver por ejemplo Conklin 1975: 21; Wallace 1979: 44; Dwyer 1979: 72, fig. 13; Doyon-Bernard 1990; Splitstoser et al. 2009; Kaulicke et al. 2009.

29 En el campo del tejido, ver, por ejemplo, Desrosiers 1996 y 2000.
} 
Figura 15: a) Borde de un paño de algodón con barra tejida con dos tramas complementarias de lana de camélido amarilla y café (@ J. Splitstoser). Fue descubierto en 2003 en Cerrillos, valle de Ica, por Mercedes Delgado y Dwight Wallace. Probable Fase 9 del Horizonte Temprano (Splitstoser en prensa). b) Diseño de la barra; la línea vertical localiza el pliegue (diseño hecho en colaboración con Jeffrey Splitstoser). El segundo borde, en la otra extremidad del paño, no se ha conservado lo bastante como para reconstruir su diseño, que era en fibra de camélido roja y café.

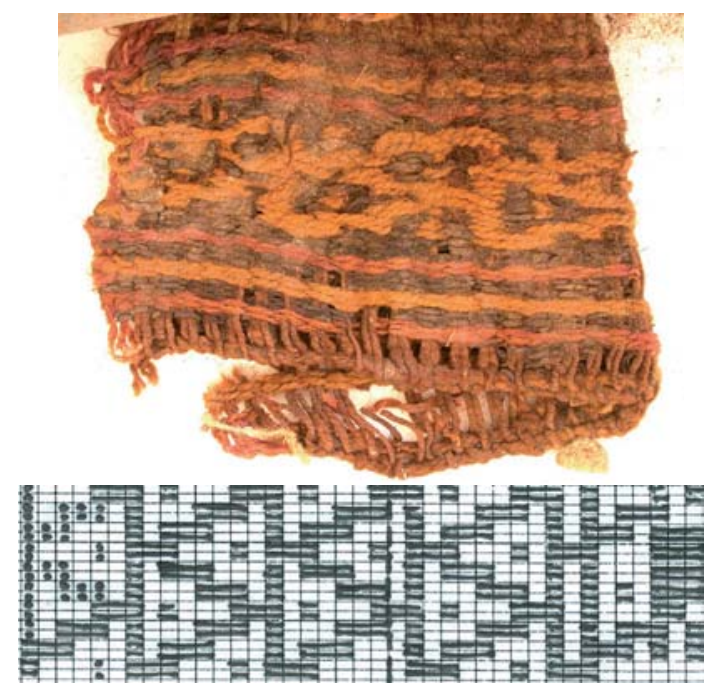

tas en Jahuay: en tejido llano con urdimbres de sustitución (King 1965: 171-174; Kajitani 1982: pl. 22; Desrosiers 2010) o en bordado con puntadas paralelas a la urdimbre (Dawson 1979: fig.24: Wallace 1979: fig.10-12) ${ }^{30}$. Esos objetos parecen responder a la voluntad de imitar no solamente el diseño sino también la forma de las bandas tejidas con urdimbres complementarias.

El último ejemplo es la túnica abierta, del mismo museo, en tejido llano con urdimbres y tramas discontinuas que tiene el diseño de serpientes entrelazadas de la Figura $14 \mathrm{c}$ re-interpretado con cinco colores y en tamaño mucho mayor. No insistiremos en este caso ya publicado en otro artículo disponible en Internet (Desrosiers 2008) y daremos una mirada a lo que nos pueden contar unos textiles un poco más recientes y conservados en gran número.

\subsection{Los bordados de la Necrópolis de Paracas, Costa Sur del Perú, fin del Horizonte Temprano-Inicio del Periodo Intermedio Temprano (ca. 150 a. C.-200 d.C.)}

Los extraordinarios bordados encontrados en los años veinte por Tello en la Necrópolis de Paracas incluyen un grupo llamado de «estilo lineal» que ya ha sido comparado varias veces con tejidos de urdimbre y trama, tanto por sus diseños muy geométricos compuestos por líneas oblicuas, horizontales y verticales de varios colores (Bird y Bellinger 1954: 58; Dwyer 1971: 86; Kubler 1975: 309), como por la manera de hacer las puntadas y construir los diseños «línea por línea como si estuvieran tejidos» (Paul 1985: 95). El artículo citado de Anne Paul está lleno de observaciones muy claras que indican la visión muy aguda de nuestra colega y amiga y ella hubiera entendido todo si sus pasos la hubieran llevado a comprender cómo se teje con urdimbres complementarias.

30 King (1965: 171) describe los primeros como tejidos «con urdimbres complementarias» e insiste sobre la similitud de sus diseños con los bordados. Ver también ejemplos del Textile Museum con urdimbre de sustitución -91.937, 91.1014, 91.1034- o bordados -91.1020, 91.1021, 91.1033, 91.1036, 91.1041-. 
Empezaremos explicando que no solamente los bordados de la Necrópolis de Paracas de «estilo lineal» fueron inspirados por tejidos con urdimbres complementarias -del estilo que hemos también denominado «lineal» porque respeta las mismas reglas de construcción-, sino también la mayoría, si no todos, los bordados ejecutados con puntadas paralelas orientadas a lo largo de los bordes. Es decir, que debe ser también considerada una parte importante de los bordados del estilo «con líneas anchas»-así llamado por Ann Paul porque «una sola línea ancha de un mismo color reemplaza las numerosas finas líneas empleadas para definir las formas en el estilo lineal» (Paul 1982: 263) ${ }^{31}$-.

Otra observación básica es que la organización de esas puntadas da una textura al bordado muy similar a la textura creada por los hilos flotantes en los tejidos cara de urdimbre. Es decir que, al menos en los primeros bordados de estilo «lineal» y «de líneas anchas», las artistas-bordadoras han reproducido con sus puntadas los hilos flotantes de los tejidos llegando a un tipo de bordado usualmente llamado «de puntadas contadas». Eso permite responder a la interrogante de Anne Paul en 2002 ante el empleo masivo de la técnica del bordado en los tejidos Paracas Necrópolis, «Why embroidery?»; dicha técnica conserva muy bien la textura de los modelos tejidos además de vehicular las ideas conceptuales sobre las cuales ella insiste. Esa exactitud en la reproducción de los hilos flotantes permite también, haciendo el camino inverso, reconstruir los modelos de los bordados de estilo «lineal» $\mathrm{y}$ «de líneas anchas» sin curvas (Figura 16).

Así podemos comprender que los tejidos creados con conteo por par, $2 / 2-$ con diseños no solamente de «estilo lineal» porque sus hilos flotantes aparecen como sarga 2.2 (Figura 16), sino también los de «estilo de áreas de color» porque sus hilos flotantes 3.1 aparecen en pares alternantes (Desrosiers 2010: fig.12)- han interesado a las bordadoras de esas obras de arte. La observación de este último grupo demuestra que, hace dos mil años, usaban una manera adicional de pasar de un color a otro gracias a la inversión de dos colores (Desrosiers 2010: 3 y fig.8-III), lo cual produce elementos verticales en las formas de los diseños y nos lleva a definir un cuarto estilo que favorece las direcciones de la urdimbre y de la trama, y que proponemos llamar «estilo ortogonal» $»^{32}$.

Las bordadoras se interesaron también por los tejidos concebidos con conteo impar derivado, 2/1, con hilos flotantes en sarga 2.1 (Desrosiers 2012a: fig.16, 17). En algunos casos que parecen corresponder a representaciones de animales, en particular cóndores $^{33}$, la semejanza con los tejidos de los jalq'a es tal, que uno se pregunta si los saberes necesarios para crearlos han sido transmitidos de generación en generación durante más de dos mil años ${ }^{34}$, o si la lógica interna del sistema ha llevado otra vez, en

31 Paul (1982: 263) incluye en ese estilo no solamente los diseños con líneas anchas rectilíneas, sino también aquellos con líneas anchas curvas que tienen puntadas no paralelas y por ello no pueden ser considerados como interpretaciones de tejidos cara de urdimbre.

32 Hasta la fecha, no hemos encontrado ese proceso usado por tejedoras actuales. Fue usado mucho en representaciones de serpientes bordadas, en particular en el fardo 421 del MNAAHP: piezas n 84,110 y 133 .

33 Ver, en particular, Bird y Bellinger 1954: P1. XXV; ver también una representación de un ser humano (i?) vestido de plumas de la tumba 35 de Cabeza Larga en Dwyer 1979, fig. 16. Otros seres y animales del fardo 157 y de las colecciones del Museo de Gotemburgo pueden ser analizados así, y probablemente unos bordados de líneas anchas encontrados por Orefici en Cahuachi (Isla y Reindel 2007: fig. 4).

34 Es la primera hipótesis en la cual habíamos pensado en 2012. 

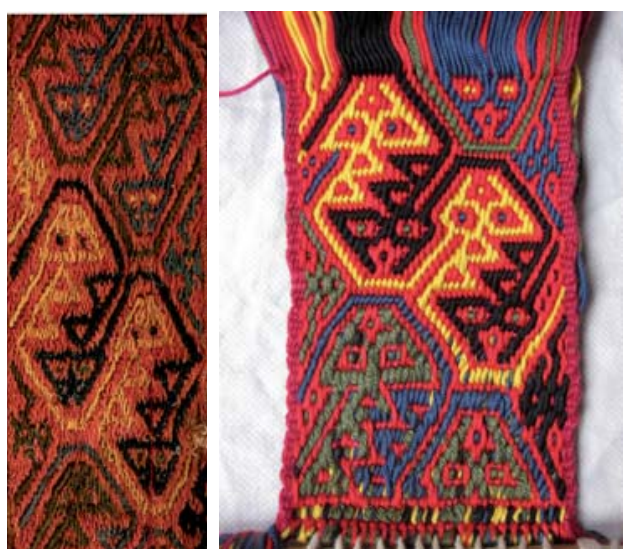

Figura 16: a) Extremo bordado del turbante 89 encontrado en el fardo 290 de Paracas Necrópolis (MNAAHP, Lima, (C) Anne Paul); b) reconstrucción del modelo tejido con 5 urdimbres y conteo por par, 2/2, «re-escogido por cantidad». (Véanse otros ejemplos en Desrosiers 2012a: fig. 17).

el siglo XIX según las investigaciones de Verónica Cereceda y Gabriel Martinez (ver nota 5), a una disposición similar de las variables con las cuales las artistas textiles conciben sus obras.

Al inicio del Intermedio Temprano se produce un cambio importante en la manera de bordar: unas artistas-bordadoras se liberan progresivamente de la dirección de la urdimbre y de la trama del tejido llano de fondo para crear puntadas en todas las direcciones y diseños poco geométricos del estilo conocido como «bloque de color». Pero el bordado con puntadas contadas paralelas continúa para otras producciones. Lo indica un tejido bordado y brocado encontrado por Strong en Cahuachi que lleva diseños de serpientes entrelazadas con marcadores muy cerca del «estilo semi-lineal» (O’Neale y Kroeber 1930: pl.1). Otra pieza, lamentablemente sin procedencia, es un muestrario o «sampler» presentando 74 figuras, la mayoría del estilo «bloques de color» $\mathrm{y}$, en una esquina, 8 diseños bordados con una organización geométrica de sus puntadas que produce los marcadores y las líneas oblicuas a los cuales estamos ahora acostumbrados (Bird 1961: fig. 2; Sawyer 1997: 77). Sin embargo, sería necesaria una observación directa de esas piezas para precisar cómo fueron creados sus diseños.

\subsection{Imitaciones de los diseños creados con urdimbres complementarias en la Costa} Central del Perú: los diseños del estilo «interlocking» o Playa Grande de la cultura Lima, Periodo Intermedio Temprano (ca. 200-650 d.C.)

En la Costa Central no se han encontrado tejidos conservados en grandes cantidades, como en la Costa Sur, para épocas tempranas. Pero diseños geométricos creados para tejidos se observan de manera repetitiva en otras obras de arte del estilo Playa Grande de la cultura Lima: la cerámica polícroma, los largos murales de Cerro Trinidad y de Cerro Culebra, mates pirograbados y un poste de madera encontrado en Playa Grande (Figura 17) ${ }^{35}$. Los peces o serpientes entrelazados característicos de la cerámica del estilo Playa Grande, predominante en las fases 4 a 6 de la seriación de

35 Para ejemplos de estilo semi-lineal: Bonavia 1985: 35-42, fig. 20-22, 25; Escobedo y Goldhausen 1999, fig. 3-5, 11-13 y nota 5; Falcón 2000, 2003; Goldhausen 2004: fig. 6 y 7; Jijón y Caamaño 1949: fig. 27; Kroeber 1926: fig. 10-11, 15-18, Makowski y Rucabado 2000: 231, fig. 56 y 58. En unos casos, el diseño muy 
Patterson (1966: 98-104), eran ya considerados por Uhle (1926: 299) como derivados del textil. Y efectivamente, a pesar de que la pintura en cerámica y muros no fue ejecutada siguiendo los hilos uno después del otro, como en los bordados de Paracas Necrópolis, pero con líneas y áreas de color bastante homogéneas, los diseños son tan característicos del «estilo semi-lineal», con sus marcadores del mismo color y a distancia establecida de las líneas oblicuas y horizontales, que no hay mucha duda sobre su origen. Como lo hemos visto arriba, los dos estilos están relacionados con tejidos cara de urdimbre creados con dos o más urdimbres complementarias y el conteo por par, 2/2 -como se puede comprobar en las reconstrucciones tejidas (Figura 18) ${ }^{36}$.

Hasta la fecha, no hemos encontrado ejemplares de estilo Lima tejidos con urdimbres complementarias, pero la orientación de la mayoría de los diseños pintados insiste sobre la disposición vertical de los hilos flotantes de los modelos, que es visible en el ángulo agudo de sus líneas oblicuas y en las reproducciones tejidas. Esa orientación es aún más visible en los cuatro lados del Poste Sagrado de Playa Grande (Figura 17c), que parece reproducir cuatro largas bandas tejidas cara de urdimbre comparables, como lo hace Falcón (2003: nota 15), con bandas en estilo lineal bordadas a lo largo de numerosas piezas de la Necrópolis de Paracas (Figura 16). Notamos también que los colores repetitivos de los diseños pintados -negro para los contornos, blanco, beige-amarillo, marrón-rojizo y café para las áreas de color- forman una gama de colores correspondiente a los colores naturales de las fibras de camélido y nos preguntamos si esa gama depende de los colores disponibles para los ceramistas o si está en relación con modelos textiles.

En el campo textil, el diseño de serpientes entrelazadas y otros diseños del «estilo semi-lineal» fueron creados localmente con muchos más colores en técnica de tapiz y probablemente en teñido a reserva del tipo anudado (o plangi) ${ }^{37}$. El fragmento de tapiz encontrado en Cerro Culebra por Mogrovejo tiene 6 colores y su diseño es muy parecido al de serpientes entrelazadas grabado en el lado A del Poste Sagrado de Playa Grande, así como a los diseños pintados en botellas y en otras cerámicas, a veces con deformaciones muy visibles (p.e., Kroeber 1926: fig. 10-11; Falcón 2003: fig. 1).

Fueron también encontrados en diferentes sitios tejidos con tramas complementarias de los cuales al menos 5 fragmentos con diseños de serpientes entrelazadas han sido publicados ${ }^{38}$. Tres de ellos tienen una particularidad hasta ahora poco con-

simplificado se vuelve lineal: Goldhausen 2004: fig. 8-10; Kroeber 1926: fig. 13; Strong y Corbett 1943: 71 y fig. 13hk y 17abcf; Willey 1943: pl. 6bd.

36 Ver también Desrosiers 2012a, fig. 19. Una reconstrucción tejida del panel 3 del mural de Cerro Culebra (Figura 17b) está también proyectada.

37 Existirían 13 fragmentos en técnica de tapiz con diseños de «estilo semi-lineal»: tres descritos por Jijón y Caamaño (1949: 408-409, 415, lám. LXXII-7, LXXIX-11, LXIV-18); cuatro por Wallace (1954), dos por Harner (1979), uno por Mogrovejo (1995) y Fernández (1995), uno por Fernández y Falcón (2006) y dos por Marcone (2012: 99-100). Jijón y Caamaño (1949: 414, lám. LXV, fig. 2) encontró en Maranga un tejido teñido por reserva con un diseño de pequeños círculos que él identifica erróneamente como batik (reserva con cera). A pesar de que la foto no permite ver claramente el fragmento, se entiende por unas cerámicas pintadas con los mismos círculos que los diseños obtenidos por reserva podían también representar serpientes entrelazadas (Kroeber 1926: 287, fig. 19; Willey 1943: lám. 8a , fig. a). Ver también Gayton 1927 con varios ejemplos en pl. 91-96, y Stumer 1953: 47 y fig. 14.

38 Uno por Wallace (1954: 137-138, fig. 92), otro por Harner (1979: 151, fig. 17-18), y tres por Fernández y Falcón (2006: fig. 11-12). Wallace (1954: 143) menciona siete «brocados» encontrados por Jijón y Caamaño 

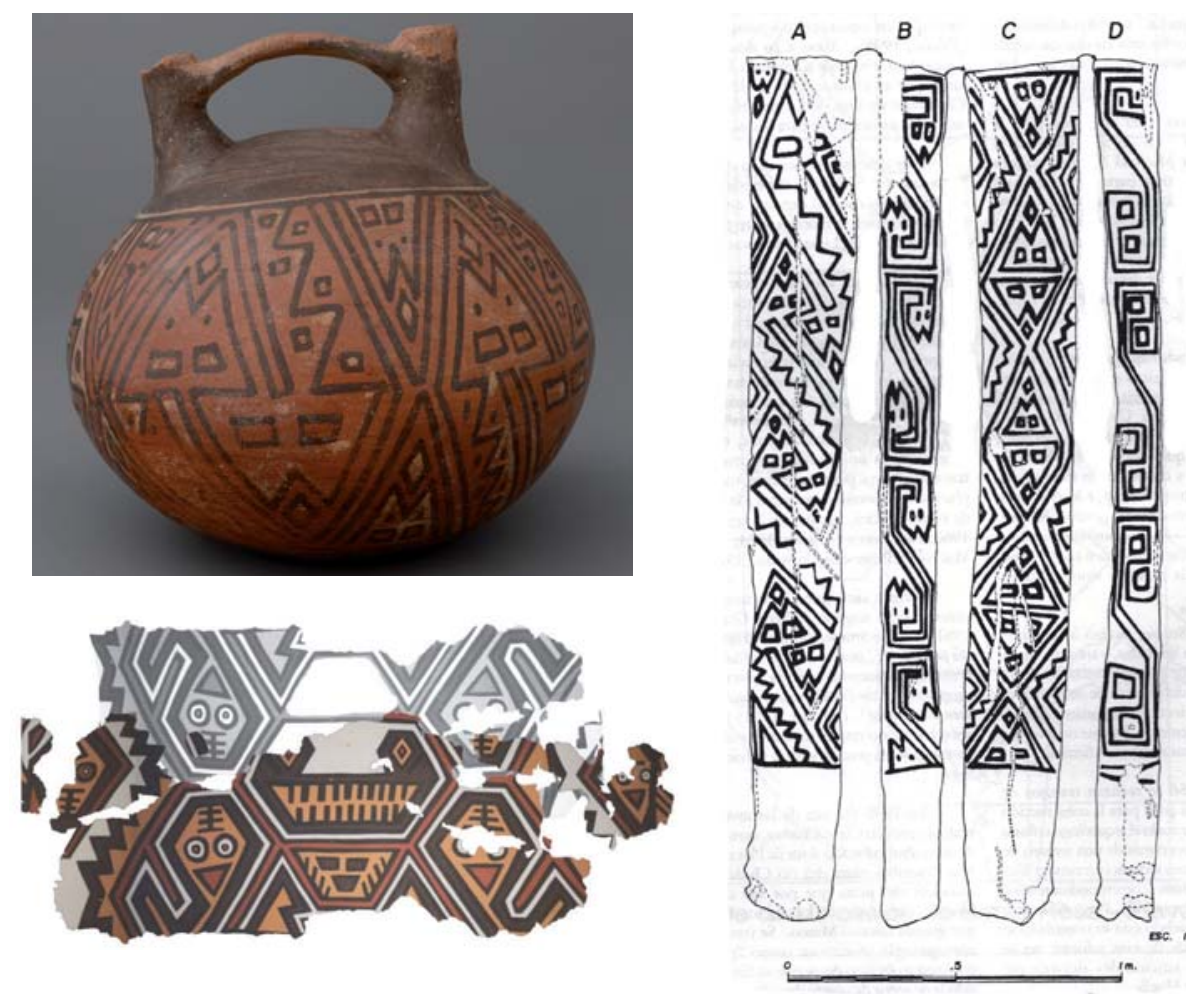

Figura 17: a) Botella de estilo Lima medio procedente de Santa Rosa - Playa Grande con serpientes entrelazadas (MNAAHP, Lima, inv. $\left.\mathrm{N}^{\circ} \mathrm{C}-18175\right)$; b) Panel 3 del mural de Cerro Culebra, valle de Chillón (Paredes 1992, (C) Paredes); c) Poste Sagrado encontrado en Playa Grande (Falcón 2000, 2003, (C) Falcón).
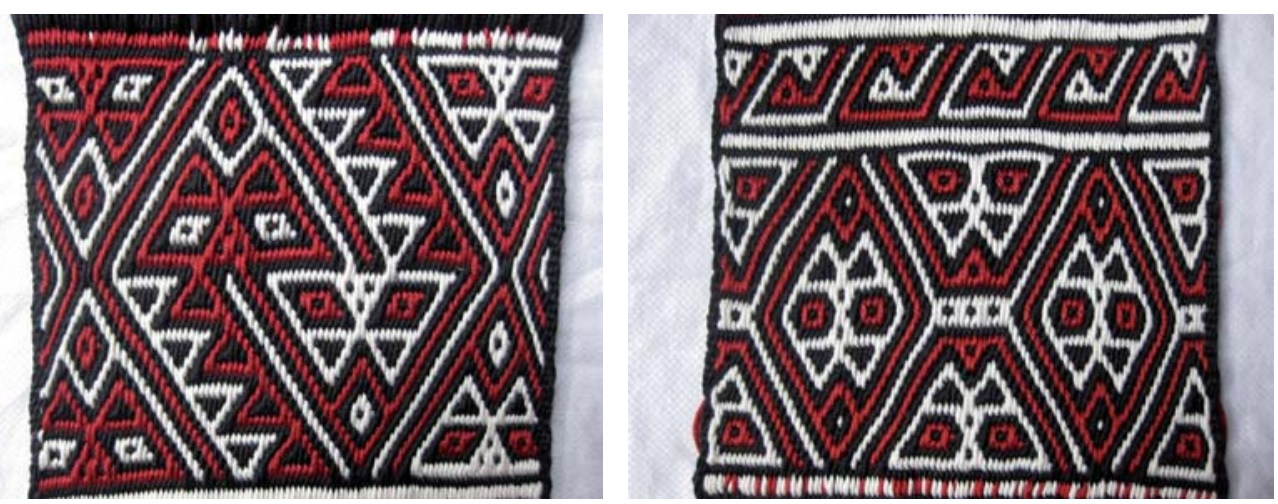

Figura 18: Reconstrucciones tejidas con 3 urdimbres complementarias de los diseños: a) de la botella de la Figura 17a; b) de un cuenco de la misma procedencia (MNAAHP, inv. $\left.\mathrm{N}^{\circ} \mathrm{C}-60934\right)$. Estos diseños son muy parecidos a los que aparecen en las caras A y C del poste sagrado (Figura 17d) y en el tapiz de Cerro Culebra (Mogrovejo 1995: fig. 5). 
siderada: a pesar de tener 3 tramas, son de doble cara. El perfil del tejido publicado por Wallace (1954: fig. 92) enseña cómo el artista ha conseguido esconder la tramas temporalmente inútiles entre las dos caras: con la trama café que aparece tanto en una cara como en la otra, y las tramas blancas y gris que se entrelazan en tejido llano con la urdimbre cuando no son necesarias para formar el diseño. La lógica empleada es bastante parecida a la practicada por los jalq'a cuando tejen con tres urdimbres (ver arriba y Figura 8). A pesar de que los perfiles cara de urdimbre y cara de trama son diferentes, porque no se resuelve el problema de la misma manera con urdimbres y con tramas complementarias, podemos preguntarnos si los modelos cara de urdimbre no eran de doble cara en muchas ocasiones.

Estas comparaciones llevan a preguntarse, como han hecho muchos otros colegas, sobre el tipo de influencia que ha podido llegar de la Costa Sur, donde el diseño de serpientes entrelazadas se encuentra con varios siglos de anticipación. Pero las diferencias entre el estilo preferido en la Costa Sur -mayormente el «estilo lineal»- y el estilo repetido en la Costa Central -mayormente el «estilo semi-lineal»-, al menos por lo que hemos podido estudiar hasta ahora, y la idea de que los tres ejemplares de bandas con urdimbres complementarias de la Figura 14 hubieran sido tejidas por tejedoras de las tierras altas, nos hace preferir la hipótesis de unas influencias serranas. Y como los estilos y los colores de las bandas permiten diferenciar las bandas con 2 y 3 urdimbres (Figura 14a y b), de la banda con 4 urdimbres (Figura 14c), nos preguntamos si no venían de dos tradiciones textiles diferentes: una al alcance de las dos zonas costeras, y la otra más en relación con la Costa Sur, o más viva en época temprana.

Otros, incluso Jijón y Caamaño (1949: 419-420) y más recientemente Makowski y Rucabado (2000), han insistido sobre los paralelos que se pueden hacer entre el estilo Playa Grande de la cultura Lima y varios diseños pintados en la cerámica de estilo Recuay. Desde el textil, nuestra impresión es que los que producían el arte Recuay se encontraban también influenciados por las producciones de las mismas tejedoras que los especialistas de Playa Grande: tejedoras herederas de técnicas con urdimbres complementarias desarrolladas durante al menos el Horizonte Temprano, probablemente en una parte de la Sierra Central o Sur. Lo que sustenta esta hipótesis es que las bandas con diseños estructurales de estilo Recuay que hemos podido localizar fueron tejidas en un triple tejido equilibrado, característico de las regiones norte del Perú, mientras sus diseños de serpientes entrelazadas tienen características conceptuales más relacionadas con el tejido con urdimbres complementarias que con el triple tejido ${ }^{39}$. Según Willey, Tello interpretaba esos paralelos como:

\footnotetext{
(1949: 407-422) en las capas proto-Lima de la Huaca III de Maranga y se pregunta si no habría otros «pattern weaves» no identificados en este grupo. El fragmento de Playa Grande publicado por Harner fue encontrado en superficie y descrito como de urdimbres complementarias, pero, a la luz de los ejemplares publicados por Fernández y Falcón, su diseño y sus características técnicas -algodón para lo que Harner piensa la trama y fibra de camélido para los hilos decorativos; orilla sin particularidad, entonces de trama-corresponde más bien a un tejido con tramas complementarias.

39 Bandas del Textile Museum de Washington (91.446), ésta estudiada por Cahlander (1985: fig. 2-11), del Metropolitan Museum de Nueva York (2001.172) (http://www.metmuseum.org/collections/search-the collections? $\mathrm{ft}=2001.172 \& \mathrm{mp} ;$ noqs=true), y del Cleveland Museum of Art (http://www.clevelandart.org/ search?search=1993.242).
} 
«... otra manifestación de una influencia de las tierras altas, comparable a, pero diferente de la Tiahuanacoide, que se extendió en el Callejón y en la Costa Central más o menos al mismo tiempo. Él relaciona esas influencias con un centro en las regiones de Montaña que él llama 'Mantaro'. Como diseño textil, se extendió a diferentes regiones donde fue adaptado para la decoración de cerámicas. Tal teoría es posible pero, al momento, no comprobada. Ciertamente en la costa no se encuentra un origen claro para el pez entrelazado. Trabajo en las regiones de Montaña puede iluminar este como otros problemas de la arqueología peruana» (Willey 1943: 195; traducción de la autora) ${ }^{40}$.

La teoría elaborada por Tello está ahora científicamente comprobada, pero no hemos encontrado todavía elementos decisivos sobre un lugar de origen en las publicaciones que hemos podido consultar sobre la arqueología de las regiones Wanka-Tarama-Chinchaycocha de la sierra central en el Intermedio Temprano (Parsons et al. 2000 y 2013). Sin embargo, la sierra central es reconocida como una zona de pastoreo de camélidos y el asiento de Huancayo Alto, en la sierra de Lima, como posible residencia ocasional de un grupo de origen serrano relacionado con el pastoreo (Browman 1976; Dillehay 1979: 27-28; Goldhausen 2001: 228) ${ }^{41}$. Volviendo a la situación presente de los jalq’a, y proyectándonos un milenio en el futuro, ¿será que los arqueólogos de entonces serán capaces de imaginar la complejidad, el significado social y cultural y la belleza de los tejidos que crean hoy en sus casas de adobe muy simples?

Se podría continuar nuestro recorrido de las obras de arte de las costas Central y Sur del Perú y, a partir del Horizonte Medio, también de la Costa Norte, porque tienen a menudo diseños o formas geométricas creados a partir de la práctica de tejer con urdimbres complementarias (Desrosiers 2008: fig. 6-7). El trabajo es mucho más complicado por razones de la diversidad de las estructuras que incluyen hilos flotantes, sean de urdimbre o de trama. Basta mirar los numerosos gráficos de los diseños publicados a partir de las colecciones del Museo Amano de Lima y del Museo Civico di Modena para darse cuenta de la proliferación de esos diseños, en particular, los que han sido creados con hilos flotantes de $3 / 1$ en pares alternados que corresponden al tejido por par 2/2 de las tejedoras actuales (Amano et al. 1962; Desrosiers y Pulini 1992: no menos de 10 estructuras diferentes están listadas en la columna 7 del cuadro p. 90 $)^{42}$. Basta también mirar en los mismos libros el número de tejidos producidos en técnica de tapiz, con tramas suplementarias, bordados, pintados, teñidos a reserva, etc. que fueron ornados con diseños construidos según las mismas reglas. La práctica de tejer con conteos rítmicos para producir diseños de tendencia geométrica ha tenido mucho éxito en numerosas tradiciones textiles precolombinas de la Costa y, por supuesto, de las tierras altas donde se desarrollaron originalmente esas prácticas.

\footnotetext{
40 «... another manifestation of a fundamental highland influence, comparable to, but different from, the Tiahuanacoid, which spread into the Callejón and onto the central coast at about the same time. He relates these influences to a center in the Montaña regions, which he calls 'Mantaro'. As a textile design, it was spread to a number of different regions where it was modified for pottery decoration. Such a theory is plausible if, as yet, unproved. Certainly on the coast there are no obvious origins for the interlocking fish. Work in the Montaña regions may illuminate this as well as many other problems of Peruvian archaeology».

41 Makowski (2002) insiste también sobre esa relación estrecha entre valle y tierras altas en el valle de Lurín. Dejamos para futuros trabajos una revisión más completa de los datos arqueológicos sosteniendo nuestra lectura de la iconografía interlocking, incluso su cronología.

42 Para una versión en español, ver Desrosiers 2006: 434, cuadro 3.
} 


\section{Conclusión}

Ahora es tiempo de concluir dejando muchos aspectos de la relación entre tejidos y otras producciones artísticas andinas para futuros estudios. Esperamos que nuestra demostración haya sido convincente y que, conforme a lo que escribió Lumbreras en 1977, y muchos otros autores, la creación textil pueda considerarse en varios aspectos como la matriz de una parte importante del Arte andino. Funciona al menos para tejidos que tienen diseños de formas geométricas de los cuales es más fácil seguir el rastro sobre otros textiles creados con técnicas menos constrictivas y sobre otros materiales.

Los diseños más geométricos están relacionados con ciertos conteos y equilibrios matemáticos y, en los casos aquí estudiados, con unos principios de complementariedad que hacen su producción bastante compleja y los tejidos mismos difíciles de reproducir sin aprendizaje. Entonces, se tiene que reconocer la perseverancia de generaciones de tejedoras que han hecho posible la conservación de esos saberes durante más de 2.500 años. Como ya lo he escrito en otro lugar (Desrosiers 1997), aparece como un movimiento de resistencia para no perder unas prácticas que estructuran elementos importantes de la vida social y, contrariamente a la visión que se ha construido sobre el tejido quechua y aymara del presente (Conklin 1998: 212), este no es solamente una continuación de los aspectos culturales de la tradiciones textiles precolombinas, sino también de sus invenciones técnicas más elaboradas. Para hacerlo visible, era necesario analizar a fondo el presente y encontrar las fuentes que podían conectarlo con el pasado. La primera etapa era plantear la hipótesis de que los saberes actuales merecían un examen profundo. La segunda etapa consistía en interesarse en los diseños geométricos del pasado que se encuentran en textiles así como en otros materiales, diseños geométricos que están a menudo considerados como repetitivos y menos dignos de interés que los diseños más realistas permitidos por la técnica de tapiz o la pintura sobre tela.

A pesar de que existen testigos más antiguos en la Costa Norte, en Huaca Prieta, que constituyen otro problema a resolver, nos hemos interesado en los diseños geométricos de la Costa Sur que surgen inmediatamente después de que la iconografía Chavín pierda su dominio en la fase 8 del Horizonte Temprano (Proulx 2008: 564). Se notan entonces cambios importantes en el campo textil con la aparición de nuevas técnicas, de nuevos diseños -en parte geométricos-, de una cantidad mayor de fibra de camélido (mayormente de alpaca y de vicuña según Renneberg et al. 2009) y por tanto importada de las alturas, y de muchos colores (King 1965: 104, 1969; Paul 1990a, 1990b). Muchos de esos cambios parecen relacionados con unos contactos con gente de las tierras altas que interesan también a Isla y Reindel (2007), pero que todavía falta identificar. Parece que en este caso, como en el de la cultura Lima en la Costa Central en la época sucesiva, existían relaciones con pueblos de las alturas antes o en otra región que la relación Huari-Nasca muy fácil de identificar por expresarse en la cerámica (Parsons et al. 2000: 58, 195). Esas relaciones pasaban por el medio textil, como lo demuestra la iconografía del estilo Playa Grande. Ha llegado la hora de reconstruir los tejidos que componían al patrimonio antiguo de sociedades de las tierras altas y de tenerlos en cuenta, a pesar de que se han conservado muy pocos. No nos parece posible continuar reconstruyendo la historia de los pueblos andinos sin hacer el esfuerzo de interesarse en los ancestros de los tejidos actuales que 
probablemente, por su alta complejidad, eran considerados también como tejidos de prestigio (Desrosiers 2012a).

Así pues, se está entendiendo poco a poco que los famosos tejidos de cumbi o compi, que tenían un papel religioso y político tan importante en el estado Inca, y que fueron considerados después de John Rowe (1946, 1979: 239) como tejidos exclusivamente en técnica de tapiz, podían haber sido tejidos también con urdimbres o tramas complementarias ${ }^{43}$. Al menos es lo que indica un número creciente de vestidos de mujeres de alta calidad tejidos cara de urdimbre con urdimbres complementarias: por ejemplo el aqsu que llevaba la mujer de Atahualpa y otro muy similar encontrado como ofrenda en el templo de Pachacamac (Acosta y Phipps 2011; Phipps 2004: 130).

Por el momento queremos solamente insistir sobre la importancia de estudiar en detalle esos tejidos raramente considerados como dignos de interés. Son la clave para entender mejor el desarrollo de las artes plásticas en los Andes Centro-Sur y, especialmente, las fases de fascinación que generaron en muchas culturas andinas sus diseños tan estilizados, de apariencia simple pero de construcción muy elaborada. Además de iluminar unos aspectos de las relaciones entre sociedades de regiones diferentes, este trabajo demuestra el aporte de los estudios textiles para entender los circuitos seguidos por la iconografía, un problema que la cerámica por sí sola no puede resolver cuando se trata de diseños creados con el medio textil.

\section{Post scriptum}

En la víspera del envío del artículo a M. J. Jiménez, la editora de este dossier, fui a verificar si se podía entender algo más de los tejidos «brocados» encontrados por Jijón y Caamaño en Maranga, en niveles de la Huaca III que él considera Proto-Lima (1949: 407-422). Después de una larga navegación entre los varios elementos descritos y las fotos de calidad limitada publicadas, hemos podido identificar cuatro chuspas de fibra de camélido con diseños tejidos con dos y tres urdimbres complementarias, probablemente con un conteo por par $2 / 2$ que produjo diseños de caras simétricas con grandes manos más o menos estilizadas en «estilo semi-lineal» (Jijón y Caamaño 1949: 398-399 y 405, fig. 209-213; Figura 19a). Jijón y Caamaño (1949: 398) describe una de ellas como: «Bolsa de brocado de lana, las figuras están contorneadas de negro, la del centro es verde sobre amarillo, las dos de los lados, rojas». Lo que, con la ayuda de sus diseños, nos permite compararlas con un ejemplo probablemente posterior perteneciente a la colección Maiman (Rosenzweig 2006, cat. 215; Figura 19b). Los modelos tejidos cara de urdimbre existían, como bien lo habíamos previsto. Además, su elaboración seguía una composición con dos ejes de simetría que tiene algo que ver con la cara sonriente pintada en el mural de Cerro Culebra (Figura 17b). Stumer (1953: 48, fig. 15) encontró en la Tumba 9 del Corte 4 una cerámica modelada representando una mujer que lleva una bolsa «parecida a las que usan los indígenas peruanos hoy en día» (traducción de la autora).

43 Mi artículo de 1986 sobre el código de Murúa fue el primero en plantear dudas sobre esa interpretación limitada de la categoría cumbi. Los trabajos siguientes han integrado poco a poco tejidos de tramas complementarias (Rowe 1995-1996: 11) y ahora de urdimbres complementarias (Frame 2010: 266). 

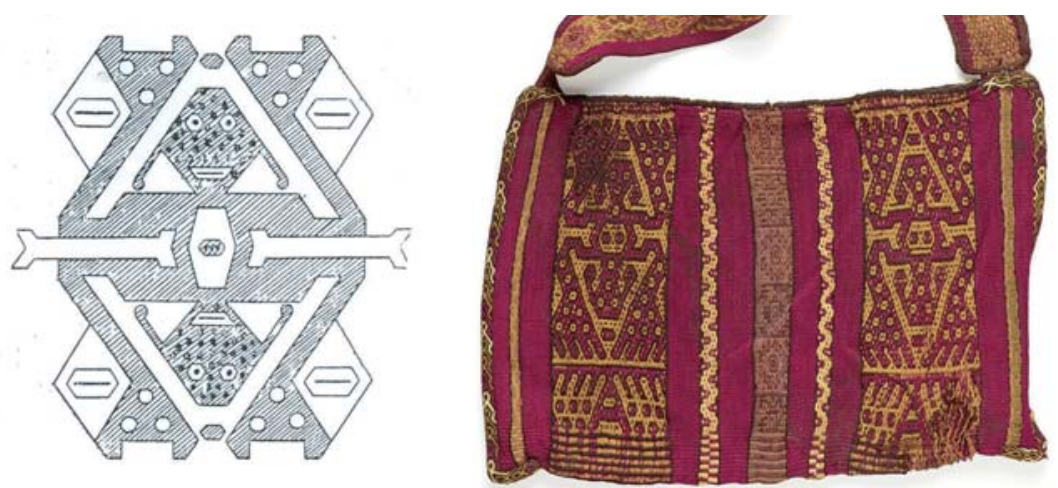

Figura 19: a) Diseño tejido con 3 urdimbres complementarias -negra, amarilla, roja- presente en una chuspa de fibra de camélido encontrada en Maranga por Jijón y Caamaño (1949: 398-399, fig. 209); b) ch'uspa con dos bandas tejidas con 3 urdimbres complementarias de los mismos colores: diseño semi-lineal con contornos negros del Horizonte Medio o fin del Intermedio Temprano; la otra cara de la bolsa tiene diseño de caras repetidas (Rosenzweig 2006, cat. 215, (C) Collección Maiman MC 749).

Las últimas líneas de este artículo se cierran con el nombre del arqueólogo que le dio inicio, L. G. Lumbreras, quien publicó en 2011 un libro sobre el trabajo de Jijón y Caamaño en Maranga, libro que esperamos leer muy pronto.

Agradecimientos: Muchas personas nos han ayudado a diferentes momentos de la investigación, sean tejedoras jalq'a como Benita y Máxima Calle en 1983 y Claudina Anagua en 2012, o colegas que nos han asistido con informaciones, bibliografía e iconografía, como Rommel Ángeles, Verónica Cereceda, Mercedes Delgado, Víctor Falcón Huayta, Arabel Fernández, Paz Núñez Requeiro, Juan Paredes Olvera, Ann Peters, Elena Phipps, Ann P. Rowe y Jeffrey Splitstoser. Queremos agradecer también las instituciones que nos han permitido estudiar sus colecciones textiles, en particular el MNAAHP en Lima, el MUSEF en La Paz, el TM en Washington D.C., el Brooklyn Museum, el AMNH y el MET en Nueva York, el BM en Londres, el Museum for World Cultures en Gotemburgo, el Völkerkunde Museum en Múnich, el Museo de América en Madrid, y el MQB en París, y a los colegas de esas instituciones, en particular Sumru Aricanli, Christine Giuntini, Anna Javér, María Ysabel Medina, Beatriz Robledo, Freddy Taboada, Carmen Thays, Ana Verde y Helen Wolfe. Contamos también con la familia de Anne Paul, que nos ha dejado trabajar con su repertorio fotográfico de los tejidos de Paracas Necrópolis, y con María Jesús Jiménez quien no solamente ha organizado este dossier sino también hecho un largo trabajo para hacer comprensible un texto escrito en un español muy aproximativo. Agradezco al EHESS por la financiación de una misión de tres meses y medio al Perú y Bolivia en 2012.

\section{Referencias bibliográficas}

Acosta, Olga y Elena Phipps

2011 «The Axsu of Atahualpa's Wife». Hali Magazine 166: 184

Amano, Yoshitaro y Yukihiro TsunOYAma

1962 Textiles of the Andes. Catalog of Amano collection. San Francisco: Heian - Tokio: Dohosha. 
Arnold, Denise Y. y Elvira ESPEJO

2012 Ciencia de tejer en los Andes: Estructuras y técnicas de faz de urdimbre. La Paz: Fundación Albó.

BARRAGÁn, Rosana

1994 «Indios de arco y flecha?»: Entre la historia y la arqueología de las poblaciones del norte de Chuquisaca (siglos XV y XVI). Sucre: ASUR.

BIRD, Junius B.

1961 «Textile Designing and Samplers in Peru», en Essays in Pre-Columbian Art and Archaeology, S. K. Lothrop et al., pp. 299-316. Cambridge: Harvard University Press.

1963a «Technology and Art in Peruvian Textiles», en Technique \& Personality, M. Mead, J. B. Bird y H. Himmelheber, eds., pp. 45-77. Nueva York: The Museum of Primitive Art.

1963b «Pre-Ceramic Art from Huaca Prieta, Chicama Valley». Nawpa Pacha 1: 29-38.

BiRD, Junius B. y Louisa Bellinger

1954 Paracas Fabrics and Nazca Needlework: 3rd century B.C.-3rd century A.D. The Textile museum, Catalogue Raisonné. Washington: The Textile Museum.

Bird, Junius B., John Hyslop y Milica D. SKInNER

1985 The Preceramic Excavations at the Huaca Prieta, Chicama Valley, Peru. Anthropological Papers of the American Museum of Natural History 62 (1). Nueva York: AMNH.

BIRRELL, Verla

1973 The Textile Arts. Nueva York: Schocken Books.

Bonavia, Ducio

1985 Mural Painting in Ancient Peru. Bloomington: Indiana University Press.

Browman, David L.

1976 «Demographic Correlations of the Wari Conquest of Junin». American Antiquity 41 (4): 465-477.

CASON, Marjorie y Adele CAHLANDER

1976 The Art of Bolivian Highland Weaving. Nueva York: Watson Guptill.

CAHLANDER, Adele

1985 Double-Woven Treasures from Old Peru. St. Paul, Minnesota: Dos Tejedoras.

Callañaupa, Nilda

2007 Weaving in the Peruvian Highlands. Cuzco: Centro de Textiles Tradicionales del Cusco.

CERECEDA, Verónica

2010 «Demonios, barroco y diseños textiles», en Entre Cielos e Infiernos. Memoria del V Encuentro Internacional sobre Barroco, T. Gisbert, ed., pp. 259-270. La Paz: Fundación Visión Cultural.

Cereceda, Verónica, Johnny DÁvalos y Jaime Majía

1993 Una diferencia, un sentido: los diseños de los textiles tarabuco y jalq'a. Sucre: ASUR.

Conklin, William J.

1975 «An Introduction to South American Archaeological Textiles with Emphasis on Material and Techniques of Peruvian Tapestry», en Irene Emery Roundtable on Museum Textiles, 1974 Proceedings: Archaeological Textiles, R. L. Fiske, dir., pp. 17-30. Washington: The Textile Museum. 
1998 «Andes», en The Extraordinary in the Ordinary, M. Hunt K., ed., pp. 209-221. Nueva York: N. Abrams.

DAwson, Lawrence E.

1979 «Painted Cloth Mummy Masks of Ica, Peru», en The Junius B. Bird Pre-Columbian Textile Conference, May 19th and 20th, 1973, A. P. Rowe, E. P. Benson y A.-L Schaffer, eds., pp. 83-104. Washington: The Textile Museum.

Desrosiers, Sophie

1986 «An Interpretation of Technical Weaving Data Found in an Early 17th Century Chronicle», en The Junius B. Bird Conference on Andean Textiles. April 7th and 8th, 1984, A. P. Rowe, ed., pp. 219-241. Washington: The Textile Museum.

1996 «Draps d'areste (III): Singularité du tissage et origine des tisserands», en Islamische Textilkunst des Mittelalters: Aktuelle Probleme, pp. 181-193. (Riggisberger Berichte 5).

1997 «Lógicas textiles et lógicas culturales en los Andes», en Saberes y memorias en los Andes, Th. Bouysse-Cassagne, ed., pp. 325-349. Lima: Institut Français d'Etudes Andines - París: Institut des Hautes Etudes sur l'Amérique Latine.

2000 «Sur l'origine d'un tissu qui a participé à la fortune de Venise: le velours de soie», en La seta in Italia dal Medioevo al Seicento. Dal baco al drappo, L. Molà, R.C. Mueller y C. Zanier, eds., pp. 35-61. Venecia: Fondazione Giorgio Cini.

2006 «Clasificaciones de las estructuras textiles y lógicas andinas», en Actas III Jornadas Internacionales sobre Textiles Precolombinos, V. Solanilla, ed., pp. 427-442. Barcelona: Universitat Autònoma.

2008 «Revisiting the Ocucaje Opened Tunic from the Textile Museum; Washington, D.C.: Textile Models and the Process of Imitation», en Textiles as Cultural Expressions. Proceedings of the $11^{\text {th }}$ Biennial Symposium of the Textile Society of America. http://digitalcommons.unl.edu/tsaconf/89/, con acceso el 30/9/2013.

2010 «Réexamen de la tunique d'Ocucaje du Textile Museum; Washington, D.C.: modèles textiles et procédés d'imitation (2e partie)». Hyper Articles en Ligne (HAL), http://hal.archives-ouvertes.fr/hal-00451774/fr/, con acceso el 30/9/2013.

2012a «Le Textil structurel». Techniques et Culture 54: 82-103.

2012b «Can We Study Textiles from Other Cultures without Ethnocentrism? The Andes as a Case Study», en Textiles and Politics. Proceedings of the $13^{\text {th }}$ Biennial Symposium of the Textile Society of America. http://digitalcommons.unl.edu/ tsaconf/674/, con acceso el 30/9/2013.

DesRosiers, Sophie y Ilaria PuLINI

1992 Tessuti precolombiani. Musei Civici di Modena. Módena: Panini.

Dillehay, Tom D.

1979 «Pre-Hispanic Resource Sharing in the Central Andes». Science 204 (4388): 24-31.

DOYOn-Bernard, Suzette J.

1990 «From Twining to Triple Cloth: Experimentation and Innovation in Ancient Peruvian Weaving (ca. 5000-400 B.C.)». American Antiquity 55 (1): 68-87.

DWYER, Jane P.

1971 Chronology and Iconography of Late Paracas and Early Nasca Textile Design. Tesis doctoral inédita. University of California, Berkeley.

1979 «The Chronology and Iconography of Paracas-Style Textiles», en: The Junius B. Bird Pre-Columbian Textile Conference, May 19th and 20th, 1973, A. P. Rowe, E.P. Benson, y A.-L Schaffer, eds., pp. 105-128. Washington: The Textile Museum. 
EMERY, Irene

1980 The Primary Structures of Fabrics. Washington: The Textile Museum.

Escobedo, Manuel y Marco Goldhausen

1999 «Algunas consideraciones acerca de la iconografía Lima». Baessler-Archiv, NF, 47: 5-37.

FALCÓN, Víctor

2000 «Playa Grande: entre la aldea y el santuario. ¿Un caso de interpretación arqueológica ambigua?». Arqueológicas 24: 53-61.

2003 «El motivo interlocking a través del ídolo de Playa Grande». Arqueológicas 26: 163-178.

FERNÁNDEZ L., Arabel

1995 «Descripción técnica de tapiz estilo 'Playa Grande'» (Apéndice de «Tapicería Lima en la Cultura Lima: Un hallazgo textil en Cerro Culebras»). Gaceta Arqueológica Andina 24: 71- 72. Lima.

FERnÁNDEZ, Arabel y Víctor FALCón

2006 «Textilería de la cultura Lima. Nuevos aportes», en $1^{a}$ Jornada sobre Estudio y Conservación de Textiles, pp. 1-23. San Miguel de Tucumán, Argentina.

Frame, Mary

2010 «Los vestidos del Sapa Inca, la Coya y los nobles del imperio», en Señoríos de los Imperios del Sol, K. Makowski, ed., pp. 260-281. Lima: Banco de Crédito.

Franquemont, Edward M.

1983 «Reserved Shed Peeble Weave in Peru», en In Celebration of a Curious Mind, N. Rogers y M. Stanley, eds., pp. 43-53. Loveland: Interweave Press.

1987 «Learning to Weave in Chinchero». Textile Museum Journal 26: 55-78.

GAYTON, Ann

1927 «The Uhle Collections from Nieveria». University of California Publications in American Archaeology and Ethnology 21 (8): 305-329. Berkeley.

Goldhausen, Marco

2001 «Avances en el estudio de la iconografía Lima». Arqueológicas 25: 223-263. Lima.

HARNER, Sandra

1979 «Early Intermediate Period Textile Sequence from Ancón, Perú», en The Junius B. Bird Pre-Columbian Textile Conference, May 19th y 20th de 1973, A. P. Rowe, E. P. Benson y A.-L Schaffer, eds., pp. 151-163. Washington: The Textile Museum.

HARris, Olivia

1978 «De l'assymétrie au triangle. Transformations symboliques au nord de Potosi». Annales, Économies, Sociétés, Civilisations 33 (5-6): 1108-1125.

IsLA, Johnny y Markus ReINDEL

2007 «Los Paracas del Sur. Una nueva perspectiva desde los valles de Palpa», en Hilos del pasado. El aporte francés al legado Paracas, pp. 79-91. Lima: Instituto Nacional de Cultura.

JiJÓN Y CAAMAÑO, Jacinto

1949 Maranga. Contribución al conocimiento de los orígenes del valle del Rímac, Perú. Quito: La Prensa Católica.

KaJITANI, Nobuko

1982 «Andesu no Senshoku [Textiles en los Andes]». Senshoku no bi - Textile Art 20: 10-96. 
Kaulicke, Peter, Lars Fehren-Schmitz, María Kolp-Godoy, Patricia Landa, Óscar Loyola, Martha Palma, Elsa Tomasto, Cindy Vergel y Burkhard Vogt

2009 «Implicancias de un área funeraria del Periodo Formativo Tardío en el departamento de Ica». Boletín de Arqueología PUCP 13: 289-322.

KING, M. Elizabeth

1965 Textiles and Basketry of the Paracas Period, Ica Valley, Peru. Tesis doctoral inédita. University of Arizona.

Kroeber, Alfred L.

1926 «The Uhle Pottery Collections from Chancay». University of California Publications in American Archeology and Ethnology 21 (7): 265-304. Berkeley.

KUBLER, G.

1975 The Art and Architecture of Ancient America: the Mexican, Maya and Andean Peoples. Baltimore: Penguin.

LUMBRERAS, Luis Guillermo

1977 «Introducción al arte textil», en Arte precolombino. Primera Parte, Arte textil y adornos, J. A. De Lavalle y W. Lang, dirs., pp. 8-23. Lima: Museo Nacional de Antropología y Arqueología.

2011 Jacinto Jijón y Caamaño. Estudios sobre Lima Prehispánica: Maranga. Quito: Instituto Metropolitano de Patrimonio Cultural.

MaKowsKi, Krzysztof

2002 «Power and Social Ranking at the End of the Formative Period: The Lower Lurin Cemeteries», en Andean Archaeology I: Variations in Sociopolitical Organization, W. H. Isbell y H. Silverman, eds., pp. 89-120. Nueva York: Plenum/Kluwer Academic.

Makowski, Krzysztof y Julio Rucabado Yong

2000 «Hombres y deidades en la iconografía Recuay», en Los dioses del antiguo Perú, pp. 198-235. Lima: Banco de Crédito.

Marcone F., Giancarlo

2012 Political Strategies and Domestic economy of the Lote B Rural Elite in the Prehispanic Lurin Valley, Peru. Tesis doctoral inédita. University of Pittsburg.

Mogrovejo R., Juan Domingo

1995 «Tapicería en la cultura Lima: un hallazgo textil en Cerro Culebras». Gaceta Arqueológica Andina 24: 63-72.

MurRA, John V.

1989 «Cloth and Its Function in the Inka State», en Cloth and Human Experience, A. B. Weiner y J. Schneider, dirs., pp. 275-302. Washington: Smithsonian Institution.

O’Neale, Lila M.

1942 «Textile Periods in Ancient Perú: II, Paracas Cavernas and the Grand Necrópolis». University of California Publications in American Archeology and Ethnology 39 (2): 143-202. Berkeley.

O’Neale, Lila M. y Alfred Kroeber

1930 «Textile Periods in Ancient Perú». University of California Publications in American Archeology and Ethnology 28 (2): 25-56. Berkeley.

Paredes O., Juan

1992 «Cerro Culebra: nuevos Aportes acerca de una Ocupación de la Cultura Lima 
(Costa Central del Perú)». Gaceta Arqueológica Andina 22: 51-62.

Parsons, Jeffrey R., Charles M. Hastings y Ramiro Matos M.

2000 Prehispanic Settlement Patterns in the Upper Mantaro and Tarma Drainages, Junin, Peru. Vol. 1, The Tarama-Chinchaycocha Region. Ann Arbor: Museum of Anthropology, University of Michigan.

2013 Prehispanic Settlement Patterns in the Upper Mantaro, Junin, Peru. Vol. 2, The Wanka Region. Ann Arbor: Museum of Anthropology, University of Michigan.

PATTERSON, Thomas C.

1966 Pattern and Process in the Early Intermediate Period Pottery of the Central Coast of Peru. Berkeley: University of California Press.

Paul, Anne

1982 «The Chronological Relationship of the Linear, Block Color, and Broad Line Styles of Paracas Embroidered Images», en Precolumbian Art History, Selected Readings, A. Cordy-Collins, ed., pp. 255-277. Palo Alto: Peek Publications.

1985 «The Stitching of Paracas Embroidered Images: Procedural Variations and Differences in Meaning». Res: Anthropology and Aesthetics 9: 91-100.

1990a Paracas Ritual Attire. Norman: University of Oklahoma Press.

1990b «The Use of Color in Paracas-Necrópolis Fabrics: What does it Reveal about the Organization of Dyeing and Designing?». National Geographic Research 6 (1): 7-21.

2002 «Why Embroidery? An Answer from the Ancient Andes», en Silk Roads, Other Roads: Proceedings of the $8^{\text {th }}$ Biennial Symposium of the Textile Society of America. http://digitalcommons.unl.edu/tsaconf/407/, con acceso el 30/9/2013.

Phipps, Elena

2004 «Garments and Identity in the Colonial Andes», en The Colonial Andes. Tapestries and Silverwork, 1530-1830, E. Phipps, J. Hecht, C. Esteras Martín, eds., pp. 17-39. Nueva York: The Metropolitan Museum of Art - New Haven: Yale University Press.

Platt, Tristan

1980 «El concepto de yanantin entre los Macha de Bolivia», en Parentesco y matrimonio en los Andes, E. Mayer y R. Bolton, eds., pp. 139-182. Lima: Pontificia Universidad Católica del Perú.

Proulx, Donald A.

2008 «Paracas and Nasca: Regional Cultures on the South Coast of Peru», en Handbook of South American Archaeology, H. Silverman y W. H. Isbell, eds., pp. 563-585. Nueva York: Springer.

RenNeberg, Rebecca, Suzanne Hummel y Bernd Herrmann

2009 «The Nasca and Their Dear Creatures - Molecular Genetic Analysis of Pre-Columbian Camelid Bones and Textiles», en New Technologies for Archaeology. Multidisciplinary Investigations in Palpa and Nasca, Peru, M. Reindel y G. A. Wagner, eds., pp. 193-203. Berlín: Springer.

RosenZweig, Alfredo (ed.)

2006 Weaving for the Afterlife. Peruvian Textiles from the Maiman Collection. Herzliya: Ampal/Merhav.

Rowe, Ann P.

1977 Warp-Patterned Weaves of the Andes. Washington: The Textile Museum.

1996-97 «Inca Weaving and Costume». Textile Museum Journal 34-35: 5-53. 
Rowe, John H.

1946 «Inca Culture at the Time of the Spanish Conquest», en Handbook of South American Indians, Vol. II: The Andean Civilizations, J. H. Steward, ed., pp. 183-330. Washington: Bureau of American Ethnology, Smithsonian Institution.

1962 «Stages and Periods in Archaeological Interpretation». Southwestern Journal of Anthropology 18 (1): 40-54.

1979 «Standardization in Inca Tapestry Tunics», en The Junius B. Bird Pre-Columbian Textile Conference, May 19th y 20th de 1973, A. P. Rowe, E. P. Benson y A.-L Schaffer, eds., pp. 239-264. Washington: The Textile Museum.

SAWYER, Alan R.

1997 Early Nasca Needlework. Londres: Laurence King.

SPLitstoser, Jeffrey C.

En prensa «Practice and Meaning in Spiral-Wrapped Batons and Cords from Cerrillos, a Late Paracas Site in the Ica Valley, Peru», en Textile, Technical Practice and Power in the Andes, D. Y. Arnold y P. Dransart, eds. Londres: Archetype Publications.

Splitstoser, Jeffrey, Dwight D. Wallace y Mercedes Delgado

2009 «Nuevas evidencias de textiles y cerámica de la época Paracas Temprano en Cerrillos, valle de Ica, Perú». Boletín de Arqueología PUCP 13: 209-235.

Strong, William Duncan y John M. Corbett

1943 «A Ceramic Sequence at Pachacamac», en Archaeological Studies in Peru, 194142, W. D. Strong, G. R. Willey y J. M. Corbett, eds., pp. 27-122. Columbia Studies in Archaeology and Ethnology, vol. 1 (3). Nueva York.

STUMER, Louis M.

1953 «Playa Grande: Primitive Elegance in Pre-Tiahuanaco Peru». Archaeology 6 (1): 42-48.

Uhle, Max

1926 «Appendix. Report on Explorations at Chancay», en «The Uhle Pottery Collections from Chancay». University of California Publications in American Archeology and Ethnology 21 (7): 293-303. Berkeley.

Wallace, Dwight T.

1954 «Appendix II: Proto-Lima Cloths from Maranga», en Proto-Lima: A Middle Period Culture of Peru, A. L. Kroeber, ed., pp. 127-148. Chicago: Chicago Natural History Museum.

1979 «The Process of Weaving Development on the Peruvian Coast», en The Junius B. Bird pre-Columbian Textile Conference, A. P. Rowe, E. P. Benson y A.-L. Schaffer, eds., pp. 27-50. Washington: The Textile Museum.

WILLEY, Gordon R.

1943 «Excavations in the Chancay Valley», en Archaeological Studies in Peru, 194142, pp. 124-196. Nueva York: Columbia University Press. 